OPEN ACCESS

Edited by:

Roberto Tuberosa

University of Bologna, Italy

Reviewed by:

Angelica Giancaspro,

University of Bari Aldo Moro, Italy

Filippo Maria Bassi,

International Center for Agricultural

Research in the Dry Areas

(ICARDA), Morocco

${ }^{*}$ Correspondence:

Bianyun Yu

Bianyun.Yu@nrc-cnrc.gc.ca

'Present address:

Asheesh K. Singh,

Department of Agronomy,

lowa State University, Ames,

IA, United States

Ron DePauw,

Advancing Wheat Technologies,

Swift Current, SK, Canada

Andrew Sharpe,

The Global Institute for Food Security, Saskatoon, SK, Canada

Specialty section:

This article was submitted to Plant Breeding,

a section of the journal

Frontiers in Plant Science

Received: 15 September 2019

Accepted: 04 February 2020

Published: 04 March 2020

Citation:

Ruan $Y, Y u B, K$ nox RE, Singh $A K$

DePauw R, Cuthbert R, Zhang W,

Piche I, Gao P, Sharpe A and Fobert P

(2020) High Density Mapping of

Quantitative Trait LoCi

Conferring Gluten Strength in

Canadian Durum Wheat.

Front. Plant Sci. 11:170.

doi: 10.3389/fpls.2020.00170

\section{High Density Mapping of Quantitative Trait Loci Conferring Gluten Strength in Canadian Durum Wheat}

\author{
Yuefeng Ruan ${ }^{1}$, Bianyun $\mathrm{Yu}^{2 *}$, Ron E. Knox ${ }^{1}$, Asheesh K. Singh ${ }^{1 \dagger}$, Ron DePauw ${ }^{1 \dagger}$, \\ Richard Cuthbert ${ }^{1}$, Wentao Zhang ${ }^{2}$, Isabelle Piche ${ }^{1}$, Peng Gao ${ }^{2}$, Andrew Sharpe ${ }^{2 \dagger}$ \\ and Pierre Fobert ${ }^{3}$ \\ 1 Swift Current Research and Development Centre, Agriculture and Agri-Food Canada, Swift Current, SK, Canada, \\ ${ }^{2}$ Aquatic and Crop Resource Development, National Research Council Canada, Saskatoon, SK, Canada, ${ }^{3}$ Aquatic and \\ Crop Resource Development, National Research Council Canada, Ottawa, ON, Canada
}

Gluten strength is one of the factors that determine the end-use quality of durum wheat and is an important breeding target for this crop. To characterize the quantitative trait loci (QTL) controlling gluten strength in Canadian durum wheat cultivars, a population of 162 doubled haploid (DH) lines segregating for gluten strength and derived from cv. Pelissier $\times$ cv. Strongfield was used in this study. The DH lines, parents, and controls were grown in 3 years and two seeding dates in each year and gluten strength of grain samples was measured by sodium dodecyl sulfate (SDS)-sedimentation volume (SV). With a genetic map created by genotyping the $\mathrm{DH}$ lines using the Illumina Infinium iSelect Wheat 90K SNP (single nucleotide polymorphism) chip, QTL contributing to gluten strength were detected on chromosome 1A, 1B, 2B, and 3A. Two major and stable QTL detected on chromosome 1A (QG/u.spa-1A) and 1B (QG/u.spa-1B.1) explaining 13.7-18.7\% and $25.4-40.1 \%$ of the gluten strength variability respectively were consistently detected over 3 years, with the trait increasing alleles derived from Strongfield. Putative candidate genes underlying the major QTL were identified. Two novel minor QTL (QG/u.spa-3A.1 and QG/u.spa-3A.2) with the trait increasing allele derived from Pelissier were mapped on chromosome $3 \mathrm{~A}$ explaining up to $8.9 \%$ of the phenotypic variance; another three minor QTL (QG/u.spa-2B.1, QGlu.spa-2B.2, and QG/u.spa-2B.3) located on chromosome 2B explained up to $8.7 \%$ of the phenotypic variance with the trait increasing allele derived from Pelissier. QG/u.spa-2B.1 is a new QTL and has not been reported in the literature. Multi-environment analysis revealed genetic $(Q T L) \times$ environment interaction due to the difference of effect in magnitude rather than the direction of the QTL. Eleven pairs of digenic epistatic QTL were identified, with an epistatic effect between the two major QTL of QGlu.spa-1A and QGlu.spa-1B.1 detected in four out of six environments. The peak SNPs and SNPs flanking the QTL interval of QGlu.spa-1A and QG/u.spa-1B.1 were converted to Kompetitive Allele Specific PCR (KASP) markers, which can be deployed in marker-assisted breeding to increase the efficiency and accuracy of phenotypic selection for gluten strength in durum wheat. The QTL that were expressed consistently across 
environments are of great importance to maintain the gluten strength of Canadian durum wheat to current market standards during the genetic improvement.

Keywords: durum wheat, gluten strength, sodium dodecyl sulphate-sedimentation volume, quantitative trait loci, single nucleotide polymorphism

\section{INTRODUCTION}

Durum wheat (Triticum turgidum L. ssp. durum), a tetraploid with $\mathrm{A}$ and $\mathrm{B}$ genomes $(\mathrm{AABB})$, is an economically important crop and the source of semolina for the production of pasta, couscous, and various types of baked products particularly in Mediterranean countries (Sapirstein et al., 2007). Global durum production reached 40.2 million metric tons in 2016 (http:// agfax.com/2017/03/23/wheat-market-global-durumproduction-expected-to-fall-in-201718/) with 7.8 million tons produced in Canada (http://www.world-grain.com/articles/ news_home/World_Grain_News/2016/12/Canada_wheat_ production_up_15.aspx?ID=\%7BD9C6D337-5F18-480D-B635E996394D6E6C\%7D\&cck=1). Gluten strength, the ability of the gluten proteins to form a satisfactory protein/starch network that promotes good cooking quality, is a key determinant of the enduse quality in durum wheat (Dexter et al., 1980). Strong gluten is a prerequisite for the production of dough with excellent rheological characteristics and hence desired quality in the finished pasta products with greater textual characteristics and increased stability to overcooking (Irvine, 1971). Gluten strength relates to the balance between viscosity and elasticity (Sissons, 2008). A positive relationship between gluten strength and low temperature dried pasta viscoelasticity has been reported (Ames et al., 2003). Strong gluten with high elastic recovery gives better cooking stability and higher cooked firmness scores (Liu et al., 1996). Rheological properties of semolina, determined by the mixograph, farinograph, extensigraph, and alveograph, are generally used to predict the cooked pasta quality (Kovacs et al., 1997). It is widely accepted that semolina from extra strong durum wheat produces firmer pasta, although the optimal level of gluten strength required for firm pasta is not clear (Sissons, 2008). Pasta quality factors of commercial importance have been the primary focus of cultivar improvement and tested for the acceptability of any new durum cultivar in Canada resulting in substantial improvement over time (Clarke et al., 2010). As such, gluten strength is an important target for genetic improvement of Canadian durum varieties.

Gluten strength variation among genotypes is mainly affected by quality and quantity of gluten proteins which are composed of polymeric glutenins and monomeric gliadins categorized by their solubility in aqueous alcohol (Autran and Feillet, 1985; Du Cros, 1987; Feillet et al., 1989; Kovacs et al., 1991; Kovacs et al., 1993). Glutenins and gliadins, together accounting for about $75-80 \%$ of total flour protein, contribute to the rheological properties of the dough (Kumar et al., 2013). Gliadins are classified as $\alpha / \beta, \gamma$, and $\omega$ gliadins according to their different mobility in an acidpolyacrylamide gel electrophoresis system [reviewed by (Barak et al., 2015)]. Glutenins can be further classified into two groups based on high and low molecular weight subunits (HMW-GS and LMW-GS) reflected by their mobility during sodium dodecyl sulfate polyacrylamide gel electrophoresis (SDS-PAGE). The HMW-GS comprise about $20-30 \%$ of the glutenin (Shewry et al., 1992; Henkrar et al., 2017). LMW-GS, the major class of glutenin subunits, accounts for $70-80 \%$ of the glutenin and a strong positive correlation of LMW-GS with durum wheat quality has been reported [reviewed by (Sissons, 2008)]. The ratio of glutenin to gliadin and the ratio of HMW-GS to LMWGS are directly related to the functional properties of the dough (Wrigley et al., 2006; Sissons et al., 2007).

Various tests were used for the prediction of gluten strength, such as SDS-sedimentation test, gluten index, alveograph, and mixograph. The SDS-sedimentation test has positive correlation with gluten strength and has been widely used for the evaluation of quality of gluten protein and for fast screening in durum wheat breeding programs due to a few advantages such as the small sample size required, simplicity, and rapidness (Dexter et al., 1980; Quick and Donnelly, 1980; Clarke et al., 1998). SDSsedimentation volume (SV) was reported to be a good predictor of cooked pasta disk viscoelasticity (Kovacs et al., 1995a) and has been widely used for evaluation of gluten strength in durum wheat breeding programs (Clarke et al., 1998). The efficacy of SV as the predictor for gluten strength might be confounded by the low to moderate positive correlation between SV and grain protein concentration (GPC) (Kovacs et al., 1995b; Clarke et al., 2010). However, no correlation between GPC and SV was reported as well (Brites and Carrillo, 2001).

Genetic studies have proposed the quantitative nature of the gluten strength trait with multiple genes coding glutenins and gliadins. Gliadins are encoded by loci Gli-1 and Gli-2 located on the short arm of the homoeologous group of chromosome 1 and 6 (Payne, 1987; Anderson et al., 2009). The Gli-B1 locus on the short arm of chromosome $1 \mathrm{~B}$ encoding $\gamma$-gliadins bands $(\gamma-45 / \gamma$ 42) was reported to be associated with gluten strength (Joppa et al., 1983; Pasqualone et al., 2015). Selection for the favorable $\boldsymbol{\gamma}$ 45 gliadin allele using a monoclonal antibody was implemented in very early generation of durum breeding (Clarke et al., 1998). However, later studies indicated that it was the linked LMW-2 rather than the $\gamma-45$ gliadin that was directly associated with gluten strength (Pogna et al., 1990). LMW-GS are encoded by gene clusters at Glu-A3 and Glu-B3 loci tightly linked with Gli-1 on the short arms of chromosome 1 (D'Ovidio and Masci, 2004).

The HMW-GS displayed a high level of polymorphism and are encoded by Glu-1 loci (Glu-A1, Glu-B1) on the long arms of chromosomes 1A and 1B (Payne and Lawrence, 1983). Each Glu- 
1 locus contains two closely linked genes encoding two different types of HMW-GS, higher molecular weight $\mathrm{x}$-type subunit and lower molecular weight y-type subunits (Shewry et al., 1992). Not all of these Glu-1 genes are expressed in certain cultivars, resulting in variation in HMW-GS subunit number between genotypes (Xu et al., 2009). The Glu-B1 locus presented higher polymorphism compared with Glu-A1. There are considerable allelic variations at $G l u-A 1$ and Glu-B1 loci and a total of 40 alleles (6 for Glu-A1 and 34 for Glu-B1) and 62 subunit combinations, were detected among 205 accessions of cultivated emmer wheat (T. turgidum ssp. dicoccum Schrank) collected from different regions of Europe and China ( $\mathrm{Li}$ et al., 2006). Similarly, a total of 43 alleles, including 5 at $G l u-A 1$ and 38 at $G l u-B 1$, resulting in 60 different allele combinations were identified in 232 accessions of durum wheat (T. turgidum L. ssp. durum) originated from various countries (Elfatih et al., 2013).

Moreover, quantitative trait loci (QTL) associated with gluten strength of durum wheat have been reported on a number of chromosomes, including chromosomes 1 and 6. Along with the major QTL on chromosome 1B and 1AL, Blanco et al. (1998) identified six additional loci on chromosomes 3AS, 3BL, 5AL, 6AL, and 7BS associated with gluten strength. As for the most quantitative traits, it has been reported that interaction among minor QTL, and between minor QTL and environment in addition to major effect QTL determine the expression of gluten strength. Patil et al. (2009) reported three major effect QTL located on chromosome 1B in proximity to glutenin coding loci Glu-B1, Glu$B 2$, and $G l u-B 3$ along with seven epistatic QTL distributed on six chromosomes (1A, 1B, 4A, 5B, 6A, and 7A) involved in four digenic epistatic interactions $(\mathrm{Q} \times \mathrm{Q})$. $\mathrm{QTL} \times$ environment $(\mathrm{Q} \times \mathrm{E})$ interactions also contributed to the variation in gluten strength (Patil et al., 2009). However, a recent study (Kumar et al., 2013) identified only one QTL consistently expressed across three environments on chromosome 1BS explaining up to $90 \%$ of the phenotypic variation and no $\mathrm{Q} \times \mathrm{Q}$ or $\mathrm{Q} \times \mathrm{E}$ interactions were observed. The differences in these studies, at least in part, could result from the different genetic background of the mapping populations. Haplotype-trait association analysis detected five loci associated with gluten index on chromosomes $1 \mathrm{~A}, 1 \mathrm{~B}, 2 \mathrm{~B}, 4 \mathrm{~B}$, and $7 \mathrm{~A}$ with the locus on $4 \mathrm{~B}$ explaining the highest amount of phenotypic variation in 192 Canadian durum wheat breeding lines (N’Diaye et al., 2018).

Reconstituting gluten strength to current market standards during genetic improvement for other traits is difficult due to the complex quantitative nature and the environmental effect on the expression of the trait. Therefore, molecular markers closely associated with QTL underlying gluten strength are of great value for developing marker-assisted selection in the durum breeding programs. In this study, we aimed to characterize genetic components controlling gluten strength in Canadian durum wheat. Along with identification of QTL, the epistatic interaction among QTL and the interactions between QTL and environmental factors, and putative candidate genes are also reported. The findings here will facilitate the marker assisted breeding for gluten strength in durum wheat.

\section{MATERIALS AND METHODS}

\section{Population and Field Trials}

A durum wheat population of 162 doubled haploid (DH) lines developed with the maize pollen method (Humphreys and Knox, 2015) and derived from Pelissier $\times$ Strongfield segregating for gluten strength was used in this study. Strongfield, selected from the cross AC Avonlea/DT665, is a registered Canada Western Amber Durum variety with strong gluten and low cadmium, developed at the Agriculture and Agri-Food Canada-Swift Current Research and Development Centre, Swift Current, SK (Clarke et al., 2005). Pelissier, a founder influencing the Canadian durum wheat gene pool, is a variety introduced from North Africa in 1929 (Dexter, 2008). It has high cadmium and lipoxygenase. The DH lines, along with their parents and controls were grown in field trials during year 2014, 2015, and 2016. Experiment was conducted as a randomized complete block design with two replicates at each of two seeding dates (early, E; late, L) and 1 week interval between two seeding dates per year. The field trial of each seeding date was grown at the different locations near Swift Current, SK, Canada. For phenotypic data analysis and QTL mapping, each different seeding date in each year was considered as one environment providing a total of six environments labeled as E14, L14, E15, L15, E16, and L16. Pre-plant soil testing was conducted each year to determine the rate of fertilizer application. The fertilizers were applied to target $112 \mathrm{~kg} \mathrm{ha}^{-1}$ nitrogen, $67 \mathrm{~kg} \mathrm{ha}^{-1}$ phosphorus, and $22 \mathrm{~kg} \mathrm{ha}^{-1}$ sulfur. The soil is naturally high in potassium and did not require additional application.

\section{Gluten Strength Measurement}

The seeds harvested from each replicate of each seeding date were subjected to gluten strength measurement. Therefore, a total of four replicates of samples from each year/location over 3 years were analyzed. The durum whole grain samples were ground on an Udy mill with $1-\mathrm{mm}$ screen at $13 \%$ moisture basis. The gluten strength was determined on $2.5 \mathrm{~g}$ samples of whole grain flour samples using the SDS-sedimentation volume (SV) method of Dick and Quick (1983) as modified by Agriculture and Agri-Food Canada (AAFC) with the addition of $25 \mathrm{ml}$ of distilled water and $25 \mathrm{ml}$ SDS solution to each sample. The higher the SV, the stronger the gluten.

\section{Statistical Analysis and Biplot Analysis of Genotype-by-Environment Interaction}

Pairwise phenotypic correlations were calculated using the Pearson's correlation coefficient in the $\mathrm{R}$ package Hmisc (version 4.2-0, http://cran.r-project.org/web/packages/Hmisc/ index.html).

Analysis of variance (ANOVA) was performed using the PROC MIXED procedure of SAS 9.3 (SAS Institute, Cary, NC, USA). In the mixed model, lines were considered as fixed effects, and years, seeding dates, line $\times$ year interactions, line $\times$ seeding date interactions, line $\times$ year $\times$ seeding date interactions, seeding dates nested in years, and replications nested in years and 
seeding dates were considered as random effects. The heritability of SV was calculated as the ratio of the genetic variance and the phenotypic variance across years using $\sigma_{\mathrm{g}}{ }^{2} /\left(\sigma_{\mathrm{g}}{ }^{2}+\sigma_{\mathrm{gy}}{ }^{2} / \mathrm{y}+\sigma_{\mathrm{gs}}{ }^{2} / \mathrm{s}+\right.$ $\sigma_{\text {gys }}{ }^{2} / \mathrm{ys}+\sigma_{\epsilon}{ }^{2} /$ rys $)$, where $\sigma_{\mathrm{g}}{ }^{2}, \sigma_{\mathrm{gy}}{ }^{2}, \sigma_{\mathrm{gs}}{ }^{2}, \sigma_{\mathrm{gys}}{ }^{2}$, and $\sigma_{\epsilon}{ }^{2}$ were estimates of line, line $\times$ year interaction, line $\times$ seeding date interaction, line $\times$ year $\times$ seeding date interaction, and residual variance, respectively, and $y, s$, and $r$ represented the numbers of year, seeding date, and replication, respectively, The repeatability of SV was calculated as the ratio of the genetic variance and the phenotypic variance of individual year using $\sigma_{\mathrm{g}}{ }^{2} /\left(\sigma_{\mathrm{g}}{ }^{2}+\sigma_{\mathrm{gs}}{ }^{2} / \mathrm{s}+\sigma_{\epsilon}{ }^{2} /\right.$ rs), where $\sigma_{\mathrm{g}}{ }^{2}, \sigma_{\mathrm{gs}}{ }^{2}$, and $\sigma_{\mathrm{e}}{ }^{2}$ were estimates of line, line $\times$ seeding date interaction and residual variance, respectively, and $s$ and $r$ represented the numbers of seeding date and replication. For the estimations of the heritability and repeatability, all effects were considered random.

Biplot analysis of genotype-by-environment interaction was performed with the GGEBiplotGUI R (R version 3.0.3) package (Frutos et al., 2014). The analysis was based on a "tester-centered $(\mathrm{G}+\mathrm{GE})$ " table and row metric preserving, without any scaling.

\section{Genotyping and Genetic Map Construction}

DNA was extracted from leaves of 2-week-old seedlings of DH lines and parents using the AutoGenprep 965 (AutoGen Inc, Holliston, MA). The Infinium iSelect Wheat 90K SNP chip was used for genotyping according to the manufacturer's protocols (Illumina). Single nucleotide polymorphism (SNP) allele clustering and genotype calling was performed with GenomeStudio v2011.1 as described by Cavanagh et al. (2013). The default clustering algorithm implemented in GenomeStudio was first used to identify assays that produced three distinct clusters expected for bi-allelic SNPs. Manual curation was performed for assays that produced compressed SNP allele clusters that could not be discriminated by the default algorithm. The accuracy and robustness of SNP clustering was visually validated. SNPs with poor clustering quality, more than $30 \%$ missing data, or segregation distortion of more than 0.35 were removed. Redundant SNPs were also removed in $\mathrm{R} / \mathrm{qtl}$ (Broman et al., 2003).

A total of 1,212 polymorphic SNP markers were used for genetic map construction in MapDisto version 2.0 software (Heffelfinger et al., 2017). Markers were classified into linkage groups based on a logarithm of odds (LOD) score threshold of 7.0 and recombination of 0.3 . Genetic distances in $\mathrm{cM}$ were estimated using Kosambi's mapping function. Markers within each group were ordered using the AutoOrder command with the Seriation II method. The marker order was refined using CheckInversion and Ripple command with the sum of adjacent recombination frequencies (SARF) option. Markers showing double recombination events were re-scored. Markers detected with genotyping errors were replaced by missing values. All calculations were repeated for new linkage groups. The markers were distributed over 25 linkage groups (LGs). LGs were assigned to chromosomes based on comparison with an existing highdensity SNP-based consensus map of durum wheat (Maccaferri et al., 2015). Parents were genotyped with the published molecular markers that discriminate glutenin and gliadin to test if they are polymorphic at these loci and to facilitate the comparative mapping.

\section{Quantitative Trait Locus Mapping}

Each different seeding date in each year was considered as one environment. Mean values for the trait from two replicates in each environment were used for the detection of QTL. Outliers of trait values were detected and removed using a Z-score transformation with a threshold of 3. QTL detection was performed using composite interval mapping (CIM) in WinQTL Cartographer v.2.5 software (http://statgen.ncsu.edu/ qtlcart/WQTLCart.htm; Wang et al., 2012). A walking speed of 1 $\mathrm{cM}$ was used. Forward regression was used for the selection of the markers to control the genetic background (control markers or cofactors) with up to five control markers. A window size of $10 \mathrm{cM}$ was used to exclude closely linked control markers at the testing site. The LOD threshold at a significance level of 0.05 for declaring statistically significant QTL was calculated by 1,000 permutations. The additive effect (a) and phenotypic variance explained by each QTL $\left(R^{2}\right)$ were estimated by CIM. The identified QTL (LOD > threshold) were automatically localized with the following parameters: minimal space between peaks = $30 \mathrm{cM}$; and minimum LOD from top to valley $=1.4$. QTL detected in different environments were considered to be the same if the confidence intervals overlapped and the trait enhancing allele was contributed by the same parent.

The digenic epistatic interactions among all pairwise combinations of QTL were analyzed with multiple interval mapping (MIM) in the WinQTL Cartographer v.2.5 software. The initial QTL model was set using the CIM results obtained in each environment. The QTL model was progressively refined by searching and testing QTL or epistasis, and re-estimating. Both main additive effects of QTL and their epistatic interactions were tested for significance using the Bayesian information criterion (BIC). Not only main QTL (QTL with statistically significant main effect) and interactions among main QTL, but epistatic QTL (QTL that has no or small main effect but statistically significant interaction effect with another QTL) interacting with the main QTL were searched.

Multiple-trait composite interval mapping (Mt-CIM) implemented in WinQTL Cartographer v.2.5 was used to test for the presence of $\mathrm{Q} \times \mathrm{E}$ interaction at the main chromosome regions affecting the target trait (Maccaferri et al., 2008; Wang et al., 2012). The value of the trait in each environment was treated as a separate trait for the common genotypes. The $\mathrm{G} \times \mathrm{E}$ (H4) hypothesis was tested. All reported QTL were designated according to the Recommended Rules for Gene Symbolization in Wheat (http://wheat.pw.usda.gov).

\section{Comparative Mapping and Projection of Quantitative Trait Locus Markers Onto the Durum Wheat Consensus Genetic Map and Onto the Reference Genomes of Durum and Wild Emmer Wheat}

QTL reported in the literature and identified in this study were projected onto the durum high-density consensus genetic map 
developed by Maccaferri et al. (2015) which includes SNP, simple sequence repeat (SSR), and diversity array technology (DArT) markers by projecting either a single marker near the QTL peak position or a pair of flanking markers within the QTL interval. The genetic linkage map and the QTLs were drawn using MapChart (version 2.3) software (Voorrips, 2002). Pairwise Spearman's rank correlation was performed in $\mathrm{R}$ version 3.3.2 to compare the collinearity of the marker order on the chromosomes of the durum consensus map and the genetic map generated in this study.

The sequences of the $90 \mathrm{~K}$ SNPs were downloaded from the Kansas State University SNP marker database (http://wheatgenomics. plantpath.ksu.edu/snp/). Sequences of SSR markers were retrieved from the GrainGenes database (https://wheat.pw.usda.gov/GG3/). Sequences of DArT markers were downloaded from https://www. diversityarrays.com/technology-and-resources/sequences. Physical map positions of SNP, SSR, and DArT markers on genomes of durum wheat cv. Svevo (Maccaferri et al., 2019) and wild emmer wheat accession Zavitan (Avni et al., 2017) were aligned using the BLAST from Durum Wheat Genome Database (http://d-data. interomics.eu) and GrainGenes database (https://wheat.pw.usda. gov/GG3/wildemmer_blast). QTL markers on the physical map of durum wheat cv. Svevo and wild emmer wheat accession Zavitan were drawn with PhenoGram software (http://visualization.ritchielab. org/phenograms/plot).

\section{Development of Kompetitive Allele Specific PCR Markers}

Firstly, several SNPs in the interval of each of the target QTL were tested for $22 \mathrm{DH}$ lines plus parents using the Kompetitive
Allele Specific PCR (KASP) primers available for the Infinium iSelect Wheat 90K SNP chip (http://www.polymarker.info/ designed_primers). Then, two closest KASP markers to each target QTL were used to genotype the population. The KASP assays were performed as described by Rasheed et al. (2016).

\section{RESULTS}

\section{Phenotypic Variation Among Doubled Haploid Population}

The gluten strength of the DH population was measured using SV. The summary statistics including mean SV values, range [minimum and maximum values, and standard deviation (SD)] are shown in Figure 1. Strongfield had significant higher SV value than Pelissier in all environments except E15 and E16 (Figure 1 and Table S1). The population had the highest mean value in environment L15 (mean $=34.0$ ) and the lowest in L16 (mean $=23.6)$ which reflects the environmental effect on gluten strength. Nevertheless, except in year 2015, no significant difference was observed for the mean SV of the population between two seeding dates. Although seeding date had no significant effect, the interaction of line by year and by seeding date was significant (Table S2). SV showed high Pearson's correlations among $\mathrm{DH}$ lines across environments ranging from $r=0.85$ to 0.92 (Figure S1). The population had the largest phenotypic variation in environment L14, as indicated by the highest standard deviation (SD) and coefficient of variation
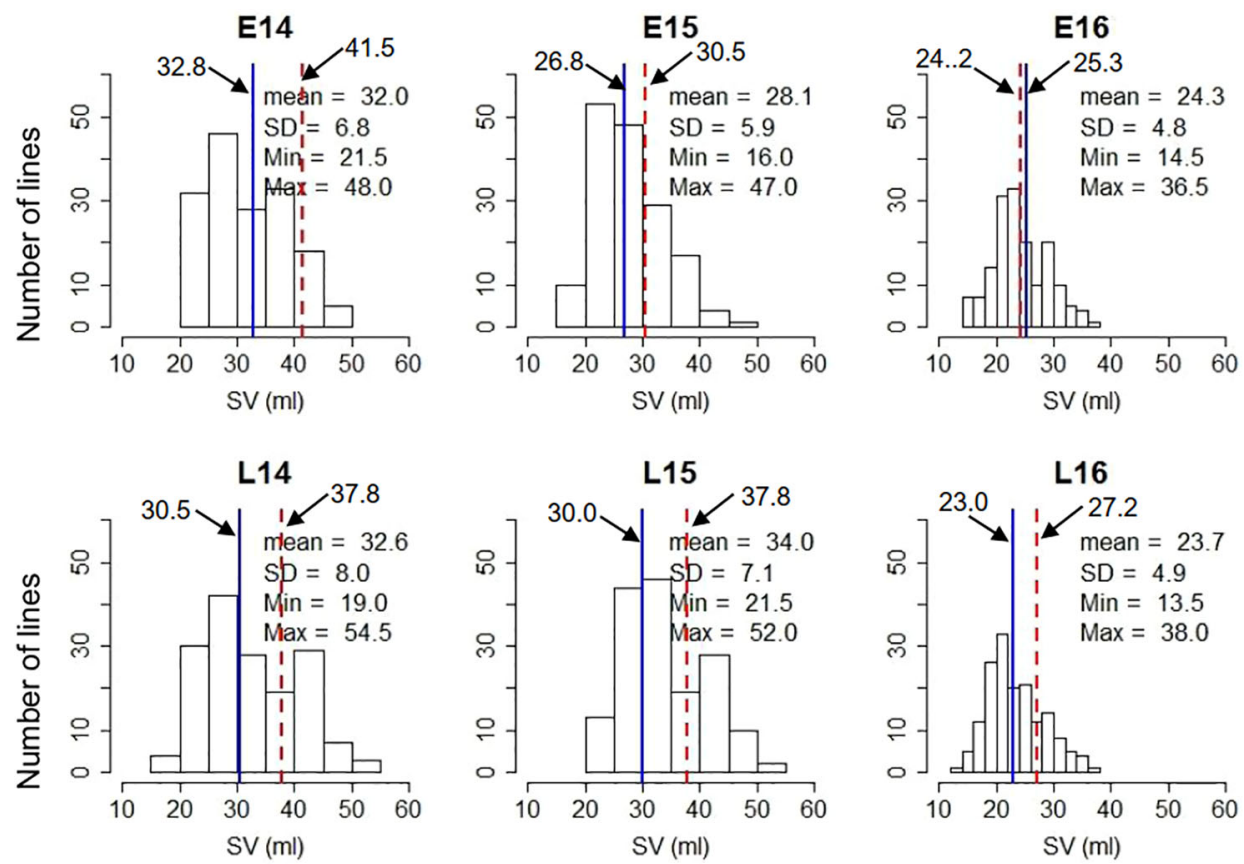

FIGURE 1 | Frequency distribution of SDS-sedimentation volume (SV) in the Pelissier $\times$ Strongfield population from 2014 to 2016 field trials with two seeding dates in each year and two replicates at each seeding date. Top panel, early seeding date; bottom panel, late seeding date. The blue solid line represents Pelissier; the red dashed line represents Strongfield; the mean SV of parents in each seeding date were shown; SD, standard deviation; Max, maximum; Min, minimum. 
$(\mathrm{CV})$, and the least variation in environment E16. Individual $\mathrm{DH}$ lines displayed bi-directional transgressive segregation, as shown by the maximum and minimum values relative to the parents (Figure 1). The transgressive segregation might result from the recombination of favorable or deleterious additive alleles from the parents, epistatic interactions of two genes, or any combinations of these mechanisms. The lines carrying favorable alleles from both parents showed higher SV than parent Strongfield, while the lines with the trait decreasing alleles from both parents had lower SV than Pelissier.

The percentages of GGE (Genotypic main effect plus Genotype-by-Environment interaction) explained by the first principal component was $90.4 \%$ and second principal component was $8.8 \%$ (Figures $2 \mathrm{~A}, \mathbf{B}$ ). The DH lines were ranked based on both mean performance and stability across environments. The single arrowed line in Figure $\mathbf{2 A}$ points to higher mean SV value across environments. Therefore, line 162 had the highest mean SV value, while line 15 had the lowest mean SV value. The AEC (average-environment coordination) ordinate (dashed line) points to the greater variability (poor stability) in either direction. Thus, lines 90 and 93 were the most stable lines across environments (Figure 2A). The position of the ideal genotype which has the highest performance in all environments, is indicated by the arrow in Figure 2B. The DH lines located closer to the ideal genotype are more desirable than others. Taken into account both mean SV and stability, line 93 was the most desirable genotype.

Significant weak to moderate positive correlation between SV and GPC was observed in three out of six environments $(r=0.3-$ 0.36) in this population (Figure S2A). In year 2014, significant correlation was shown in both seeding dates when the population had lower GPC compared to other field years. No significant correlation existed for any seeding date in year 2015. Similarly, significant weak to moderate negative correlation between SV and grain yield (GY) was also observed in the same three out of six environments $[r=-0.334-(-0.216)]$ (Figure S2B). Significant negative correlation was displayed in both seeding dates in year 2014 when higher GY was obtained. While no significant correlation was observed in the year 2015 with lower GY.

\section{Quantitative Trait Locus Mapping by Composite Interval Mapping in Single Environments}

ANOVA (Table S2) indicates that genotype by year and by seeding date interaction had significant effect on the SV. Therefore, QTL analysis was first performed for SV in each environment. Variable numbers of significant QTL, from two to five, were detected in each environment. Globally, the largest number of QTL (5) was detected in environment L14 and L15. A total of nine different QTL were detected across environments, four of which were specific for a single environment (Table 1). Both parental lines contributed the favorable alleles depending on the QTL (2 by Strongfield and 7 by Pelissier). A major QTL on chromosome 1B (QGlu.spa-1B.1) explaining up to $40.1 \%$ of the phenotypic variance $\left(R^{2}\right)$ and a second major QTL on chromosome 1A (QGlu.spa-1A) explaining up to $18.7 \%$ of the phenotypic variance, were detected across all environments with high SV allele derived from Strongfield. Two minor QTL, QGlu.spa-1B.2 and QGlu.spa-1B.3 with $R^{2}$ values of $4.1 \%$ and $6.3 \%$, were also detected on chromosome $1 \mathrm{~B}$ but only in a single environment. Additionally, three QTL on chromosome $2 \mathrm{~B}$ and two QTL on chromosome 3A were detected with $R^{2}$ values ranging from 3.4 to $8.9 \%$. The QTL QGlu.spa-3A.1 and QGlu.spa-3A.2 were repeatedly detected in at least two environments. Except QGlu.spa-1A and QGlu.spa-1B.1, no other minor QTL was detected in L16. Pelissier contributed trait-enhancing alleles to all minor QTL.
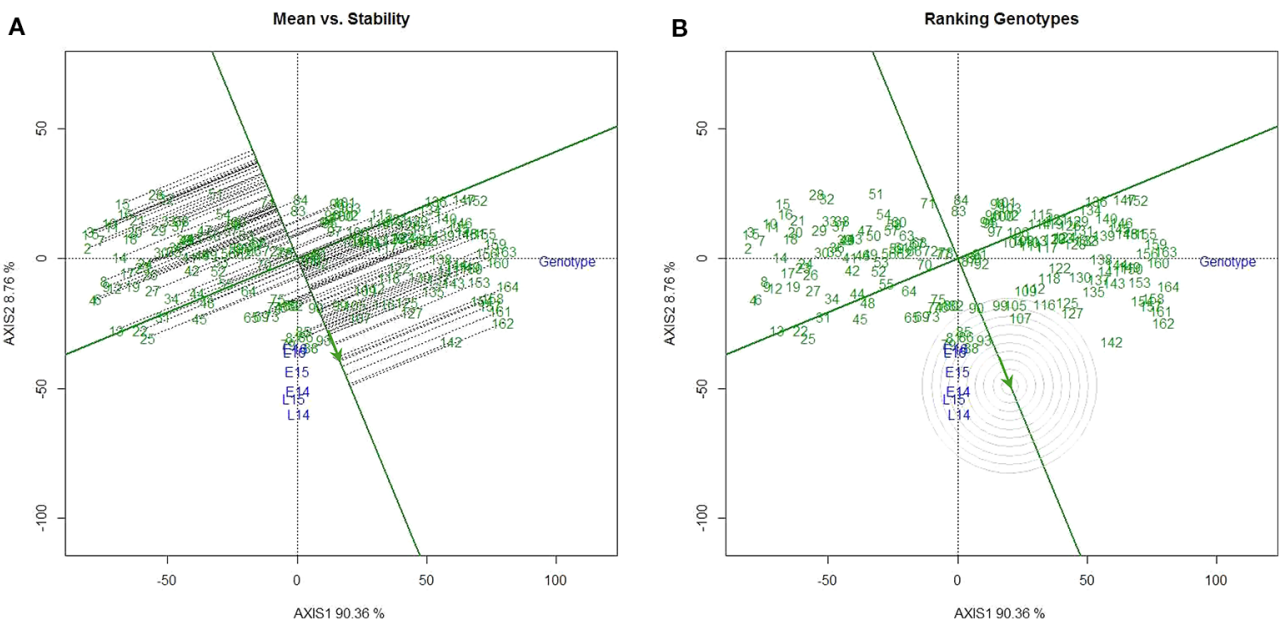

FIGURE 2 | GGE (Genotypic main effect plus Genotype-by-Environment interaction) analysis of SDS-sedimentation volume (SV) in DH lines of Pelissier $\times$ Strongfield. (A) Average-environment coordination (AEC) view of the GGE biplot; the single-arrowed line is the AEC abscissa (or AEA) and points to the higher mean SV value across environments. (B) Ranking doubled haploid (DH) lines relative to the ideal genotype (a genotype that GGE predicted has the best performance across environments for SV) on SV performance. The arrow is where an ideal genotype should be. The DH lines located closer to the ideal genotype are more desirable than others. 
TABLE 1 | Overview of quantitative trait loci (QTL) identified for SDS-sedimentation volume (SV) across six environments.

\begin{tabular}{|c|c|c|c|c|c|c|c|}
\hline Chr ${ }^{a}$ & QTL & $E_{n v}^{b}$ & Peak marker & LOD & Additive $^{c}$ & $R^{2}(\%)^{\mathrm{d}}$ & Interval (two LOD drop) \\
\hline \multirow[t]{6}{*}{$1 \mathrm{~A}$} & QGlu.spa-1A & E14 & wsnp_Ex_c13186_20822127 & 16.1 & 3.00 & 18.7 & BS00088136_51 - Kukri_c10405_1277 \\
\hline & & E15 & wsnp_Ex_c13186_20822127 & 15.1 & 2.49 & 17.6 & IAAV1142 - RAC875_c31031_387 \\
\hline & & E16 & wsnp_Ex_c13186_20822127 & 10.7 & 1.83 & 13.7 & IAAV1142 - RAC875_c31031_387 \\
\hline & & L14 & wsnp_Ex_c13186_20822127 & 14.3 & 3.09 & 14.6 & BS00088136_51 - RAC875_c31031_387 \\
\hline & & L15 & wsnp_Ex_c13186_20822127 & 18.1 & 3.14 & 18.2 & IAAV1142 - RAC875_c31031_387 \\
\hline & & L16 & wsnp_Ex_c13186_20822127 & 10.8 & 2.03 & 16.9 & IAAV1142 - RAC875_c31031_387 \\
\hline \multirow[t]{8}{*}{$1 \mathrm{~B}$} & QG/u.spa-1B.1 & E14 & Kukri_c38353_67 & 26.5 & 4.11 & 35.6 & BS00085235_51 - RCA875_rep_c74067_541 \\
\hline & & E15 & Kukri_c38353_67 & 25.6 & 3.48 & 34.6 & BS00085235_51 - RCA875_rep_c74067_541 \\
\hline & & E16 & Kukri_c38353_67 & 27.2 & 3.01 & 35.1 & BS00085235_51 - RCA875_rep_c74067_541 \\
\hline & & L14 & Kukri_c38353_67 & 22.2 & 4.71 & 25.4 & BS00085235_51 - RCA875_rep_c74067_541 \\
\hline & & L15 & Kukri_c38353_67 & 32.7 & 4.60 & 40.1 & BS00085235_51 - RCA875_rep_c74067_541 \\
\hline & & L16 & Kukri_c38353_67 & 20.4 & 2.98 & 36.5 & BS00085235_51 - RCA875_rep_c74067_541 \\
\hline & QGlu.spa-1B.2 & L14 & Excalibur_c50079_420 & 5.4 & -1.80 & 4.1 & Ku_c241_460 - BS00078029_51 \\
\hline & QG/u.spa-1B.3 & L15 & BS00067436_51 & 7.4 & -1.91 & 6.3 & Tdurum_contig7449_800 - RAC875_c47427_235 \\
\hline \multirow[t]{3}{*}{$2 \mathrm{~B}$} & QGlu.spa-2B.1 & L15 & RAC875_c38003_164 & 4.2 & -1.37 & 3.4 & Excalibur_c19499_948 - D_F5XZDLF01CFO7W_135 \\
\hline & QGlu.spa-2B.2 & E16 & Kukri_c25868_56 & 7.3 & -1.44 & 8.7 & Kukri_c25868_56 - Ex_c55735_1012 \\
\hline & QGlu.spa-2B.3 & L14 & Excalibur_c91034_141 & 5.5 & -1.79 & 5.6 & Excalibur_c33221_681 - CAP7_6910_523 \\
\hline \multirow[t]{5}{*}{$3 A$} & QG/u.spa-3A.1 & E14 & RAC875_c64107_404 & 4.1 & -1.41 & 4.0 & RAC875_c64107_404 - BS00021981_51 \\
\hline & & L15 & RAC875_c64107_404 & 4.4 & -1.40 & 3.6 & RAC875_c64107_404 - BS00021981_51 \\
\hline & QGlu.spa-3A.2 & E15 & Excalibur_c14216_692 & 5.6 & -1.46 & 5.9 & Tdurum_contig98188_239 - RAC875_c775_1264 \\
\hline & & E16 & wsnp_Ex_rep_c69864_68824236 & 6.7 & -1.42 & 8.3 & CAP_c3367_68 - Tdurum_contig98188_239 \\
\hline & & L14 & Excalibur_c14216_692 & 9.7 & -2.45 & 8.9 & Tdurum_contig98188_239 - RAC875_c775_1264 \\
\hline
\end{tabular}

${ }^{a}$ Chromosome; ${ }^{b}$ environment.

${ }^{c}$ Additive effect, the positive values indicate that the alleles from Strongfield have the effect of increasing the trait value.

${ }^{d} R^{2}$ is the percentage of phenotypic variation explained by each $Q T L$.

\section{Multi-Environment Quantitative Trait Loci Analysis}

Multi-environment QTL analysis was performed to detect the significant QTL across environments and Q $\times$ E effect (Figure 3). Two significant QTL QGlu.spa-1A and QGlu.spa-1B.1 were detected by multi-environment analysis which agreed with single-environment analysis. In these analyses, environment L15 influenced joint analysis the most for QTL QGlu.spa-1A while environment L14 influenced joint analysis the most for QTL QGlu.spa-1B.1. However, environment L14 influenced SV the least for QTL QGlu.spa-1A. Both QGlu.spa-1A and QGlu.spa$1 B .1$ were statistically significant for multi-environment joint analysis. Although the $\mathrm{Q} \times \mathrm{E}$ effect was significant, these two
QTL were stably expressed across all environments. The QTL mapped on other chromosomes in only one or two environments did not reach the significance threshold value in the multienvironment QTL analysis. Notably, the Q $\times$ E effect observed was due to the difference of the effect in magnitude and not the direction of QTL. This was also evidenced by the consistent sign of the effects of QTL detected across environments (Table 1).

\section{Implication of Quantitative Trait Loci on 1A and $1 \mathrm{~B}$ and Development of Kompetitive Allele Specific PCR Markers}

Figure $\mathbf{4 A}$ is a graphical illustration of the chromosome $1 \mathrm{~A}$ region harboring QTL QGlu.spa-1A of a selection of $20 \mathrm{DH}$

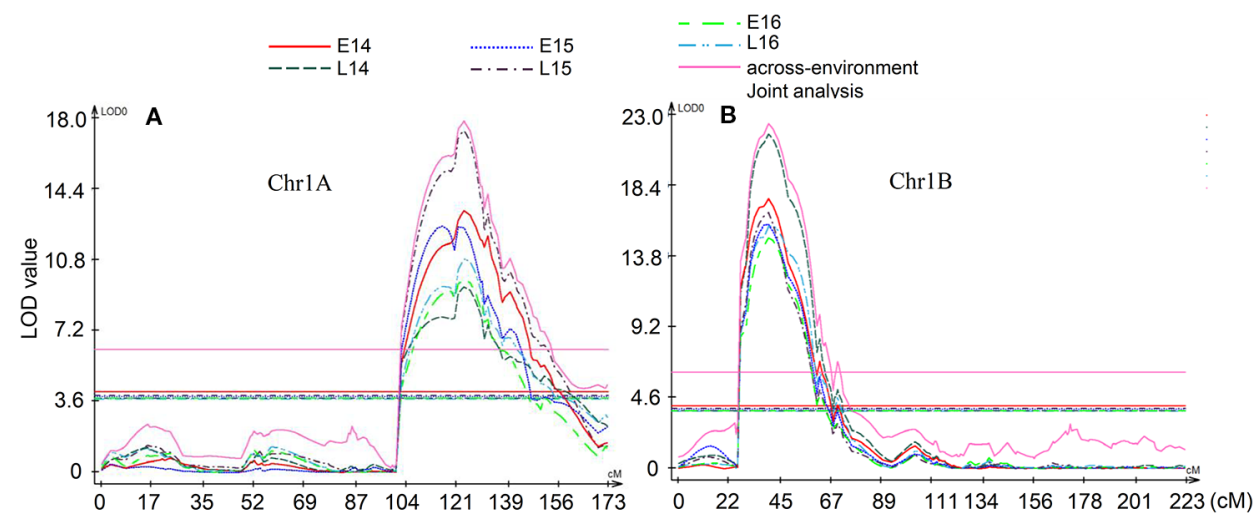

FIGURE 3 | Multi-environment quantitative trait loci (QTL) analysis. (A) QTL QG/u.spa-1A on chromosome 1A, (B) QTL QG/u.spa-1B.1 on chromosome 1B. The horizontal lines indicate logarithm of odds (LOD) significance threshold determined by 1,000 permutations at a significance level of 0.05 . 
genotypes and Figure $4 \mathrm{~B}$ of the chromosome $1 \mathrm{~B}$ region containing QGlu.spa-1B.1 for the same genotypes with high and low SV values. On both chromosome $1 \mathrm{~A}$ and $1 \mathrm{~B}$, the Strongfield alleles occurred in high SV genotypes, whereas the Pelissier alleles contributed to the low SV lines. This agreed with QTL analysis results (Table 1). Colored fragments along the chromosome region outside the green line of the peak marker, refer to the loci belonging to other traits may be not related to SV. Based on the genotypes of two flanking markers in the QTL region of QGlu.spa-1A (IAAV1142 and RAC875_c31031_387) and QGlu.spa-1B.1 (Kukri c38553_67 and RCA875_rep_c74067_541), the $\mathrm{DH}$ lines were separated into two groups with significantly different means ( $t$ test, $p<10^{-4}$ ) between the two groups (Figures 5A, B). More distinct separation was shown for the QGlu.spa-1B.1 than QGlu.spa-1A, which is in agreement with a larger portion of the phenotypic variation explained by QGlu.spa-1B.1 (Table 1). Based on the genotypes of the flanking markers of both aforementioned QTL combined, two main groups with clearer separation was observed within the population: one group of $\mathrm{DH}$ lines with high SV value (strong gluten) having flanking marker alleles from Strongfield and the other group of lines with low SV value (weak gluten) carrying flanking marker alleles from Pelissier (Figure 5C).

Two KASP assays were developed for each of QTL, QGlu.spa$1 A$ (wsnp_Ex_c13186_20822127 and IAAV1142), and QGlu.spa1B.1 (RAC875_rep_c74067_541 and Kukri_c38553_67). All KASP assays were validated against the SV values of the population in each environment (Figure 6). In all cases, the genotypes carrying Strongfield allele had significantly higher SV than those with Pelissier allele.

\section{Combined Haplotype Analysis Across Multiple Quantitative Trait Loci}

To investigate the accumulated effects of the favorable alleles on SV across multiple QTL, the combined haplotype analysis was performed on QTL detected in two or more environments, QGlu.spa-1A, QGlu.spa-1B.1, QGlu.spa-3A.1, and QGlu.spa$3 A .2$. The SNPs in the two LOD interval of each QTL were used for haplotype analysis. A total of 11 different haplotypes (Hap1-Hap11) were identified at different frequencies, with each haplotype containing three or more DH lines (Figure 7). The $\mathrm{DH}$ lines with Hap2 has the best combination of all favorable alleles at each QTL, as evidenced by the highest mean SV across all environments. The most desirable genotype, line 93, has this haplotype. While the lines with Hap10 has the least favorable combination of the alleles from each QTL. Significant difference was observed for SV in these two haplotype groups across all environments. Significant difference in SV between Hap1 and Hap8 across all environments agreed with the effect of the QGlu.spa-1A. Likewise, the significant difference in SV between Hap1 and Hap4, Hap2 and Hap7, Hap8 and Hap10, confirmed the effect of major QTL QGlu.spa-1B.1. Except in E16, no significant difference was observed between Hap1 and Hap2. This is not surprising given the environment specific expression and minor effect of QGlu.spa-3A.1 and QGlu.spa-3A.2.

\section{Identification of Epistatic Interaction of Quantitative Trait Loci}

Multiple interval mapping (MIM) has been used for mapping multiple QTL with epistasis (Laurie et al., 2014). In this study MIM was used for identification of digenic epistatic interactions
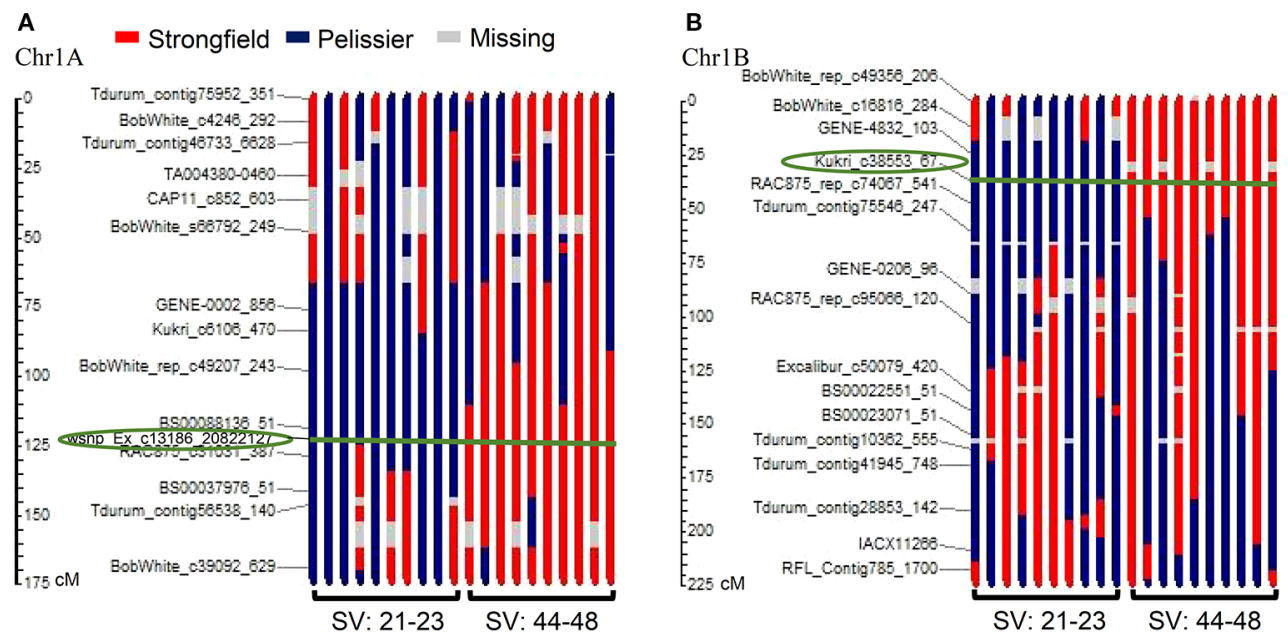

FIGURE 4 | Graphical illustration of genotypes of 20 doubled haploid (DH) lines with recombination pattern of major quantitative trait loci (QTL) (A) QGlu.spa-1A on chromosome 1A and (B) QGlu.spa-1B.1 on chromosome 1B. Circled marker is the peak marker in the QTL region and the green line indicates the peak marker position on each genotype. The blue bar represents the fragment derived from weak gluten strength parent Pelissier and the red bar represents the fragment derived from strong gluten strength parent Strongfield. SV, SDS-sedimentation volume. 

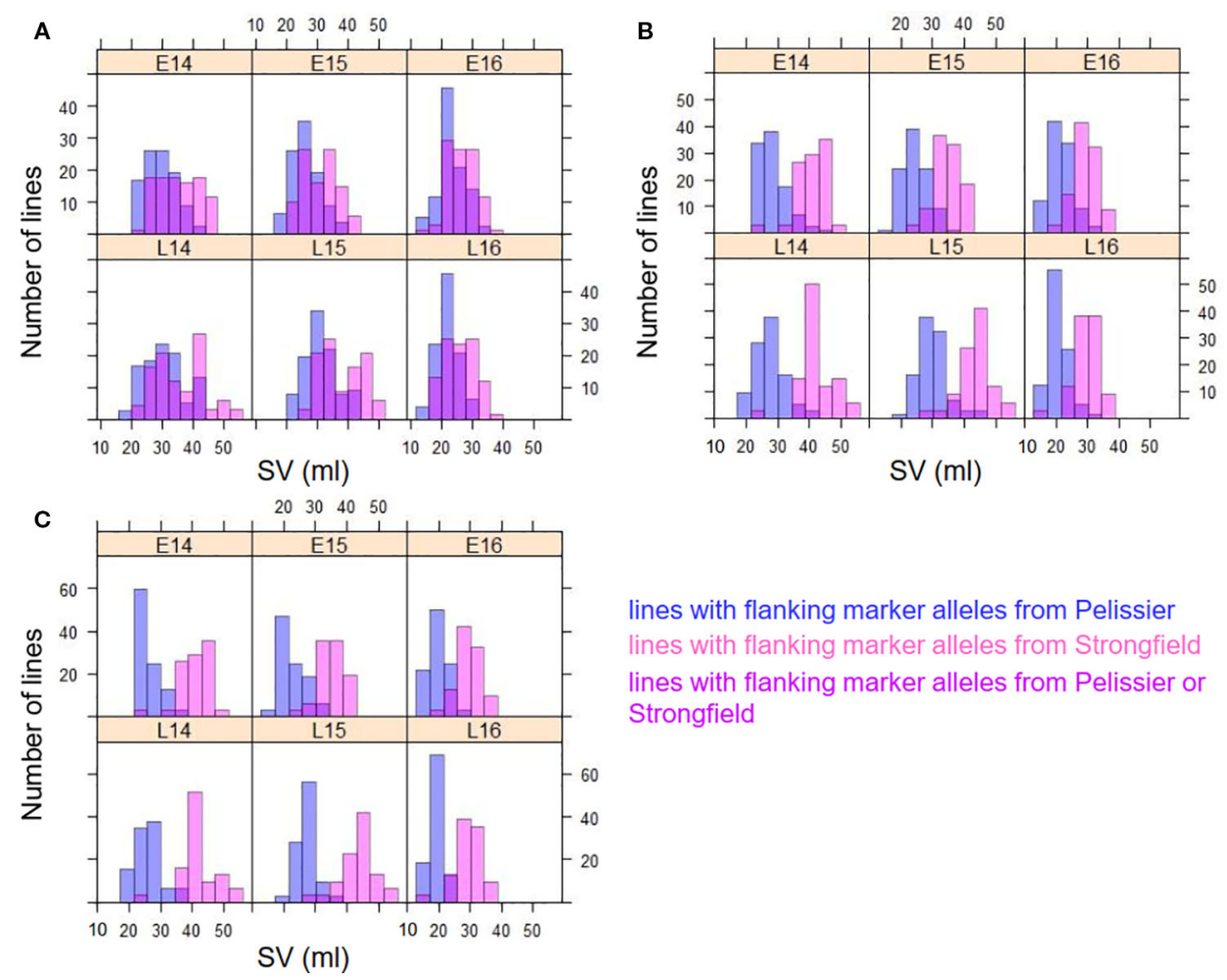

lines with flanking marker alleles from Pelissier lines with flanking marker alleles from Strongfield lines with flanking marker alleles from Pelissier or Strongfield

FIGURE 5 | Frequency distribution of SDS-sedimentation volume (SV) in two groups of DH lines separated on the genotype of two flanking markers of (A) QG/u.spa-1A (IAAV1142 and RAC875_c31031_387) on chromosome 1A, (B) QGlu.spa-1B.1 (Kukri c38553_67 and RCA875_rep_c74067_541) on chromosome 1B, and (C) both QG/u.spa-1A on chromosome 1A and QG/l.spa-1B.1 on chromosome 1B across six environments (field year 2014-2016 with two seeding dates in each year. early, E; late, L). The blue bar represents the lines with alleles from weak gluten strength parent Pelissier, the pink bar represents the lines with alleles from strong gluten strength parent Strongfield, and the magenta bar represents the lines with alleles either from Strongfield or Pelissier.

among all pairwise combinations of QTL. Compared with the results obtained by CIM analysis, two additional significant QTL, QGlu.spa-1B.4 in L14, and QGlu.spa-5A in L15, were detected with $R^{2}$ values of $9.4 \%$ and $2.3 \%$ respectively (Table 2 ). The epistatic interactions detected together with their average effects and $R^{2}$ values are reported in Table 2. A total of 11 pairwise QTL interactions (additive $\times$ additive) were detected in different environments at a significance level of 0.05 . Of note, the epistatic effect between the two major QTL QGlu.spa-1A and QGlu.spa-1B.1 was detected in four out of six environments with $R^{2}$ values of $1.4-1.9 \%$. The other 10 pairs of environment-specific interactions with $R^{2}$ values of $0.8-3.7 \%$ were detected only in a single environment. Not only interactions among main QTL (QTL with statistically significant main effect) but also epistatic QTL, QTL that has no or small main additive effect but statistically significant interaction effects with another QTL, interacting with main QTL were identified. It is interesting to note that the additive effect of QGlu.spa-5B was too small to reach the genome-wide significance level in CIM scans but it had significant interaction with identified QTL QGlu.spa-1B.4 and QGlu.spa-2B.3 in environment L14, as well as with QTL
QGlu.spa-2B.4 in L16. Likewise, epistatic QTL QGlu.spa-6B had significant interaction with other QTL in two environments, E14 and E16, but no main effect.

\section{Comparison With Previously Reported Quantitative Trait Loci}

The marker order in the genetic map generated in this study was highly collinear with the durum consensus map developed by Maccaferri et al. (2015), as indicated by the Pairwise Spearman's rank correlations ( $r=0.992-0.999)$ (Figure S4). QTL reported for SV in the literature and identified in this study were projected onto the durum wheat consensus genetic map by projecting either a single marker near the QTL peak position or a pair of flanking markers within the QTL interval (Table S3 and Figure 8). The QTL QGlu.spa-1A (peak marker: wsnp_Ex_c13186_20822127) detected in this study was projected at position $89.5 \mathrm{cM}$ on chromosome $1 \mathrm{~A}$ of the durum consensus genetic map. It is approximately $6 \mathrm{cM}$ away from SSR marker wmc312 reported to be associated with SV in durum wheat by Conti et al. (2011). The QTL QGlu.spa-1B.1 (peak SNP: Kukri_c38353_67) was projected on the short arm of chromosome $1 \mathrm{~B}$ on the durum wheat consensus 

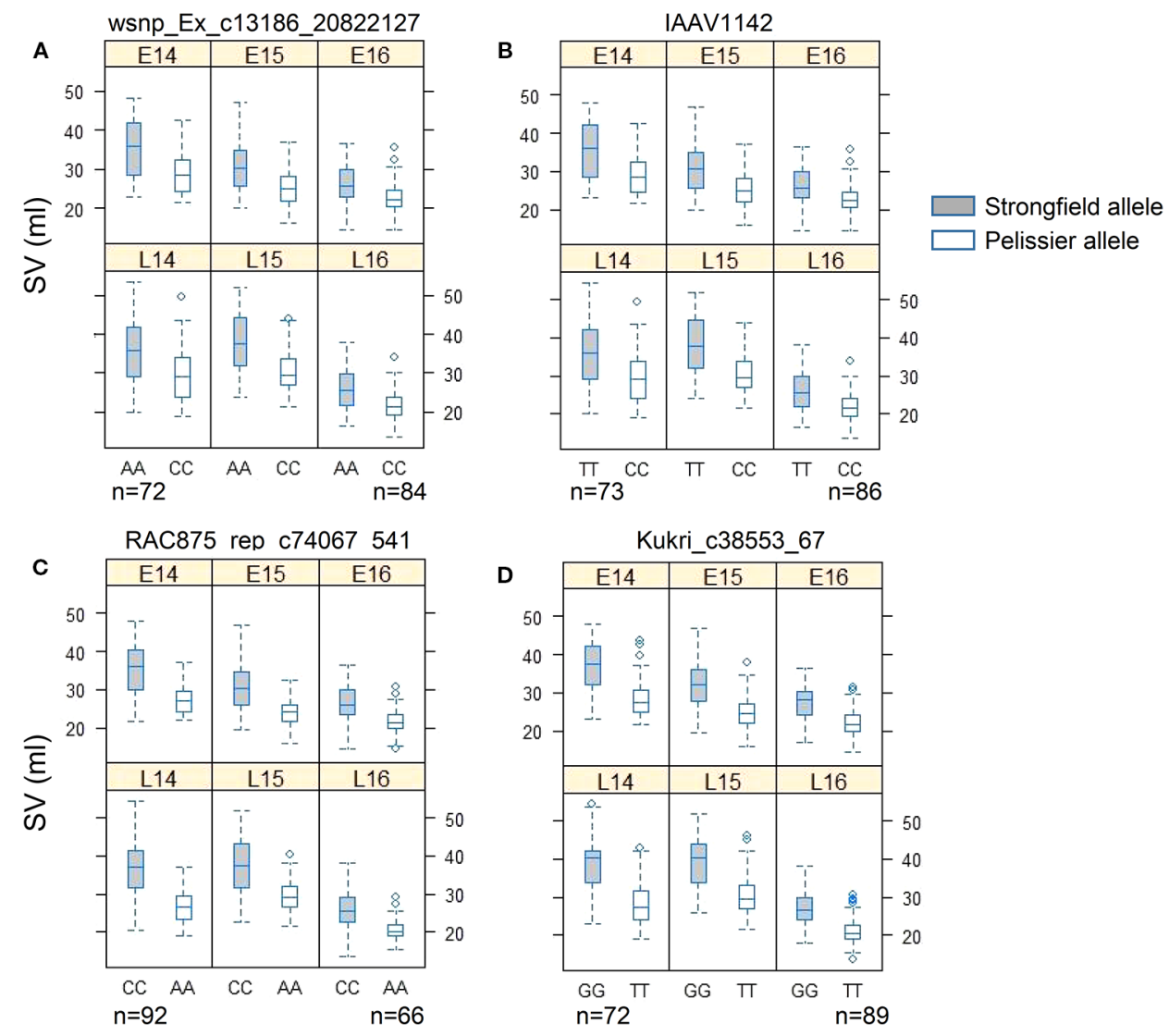

FIGURE 6 | Phenotypic validation of Kompetitive Allele Specific PCR (KASP) assays for (A) wsnp_Ex_c13186_20822127 and (B) IAAV1142 in the interval of QGlu.spa-1A on chromosome 1A, (C) RAC875_rep_c74067_541, and (D) Kukri_c38553_67 in the interval of QG/u.spa-1B.1 on chromosome 1B across six environments (field year 2014-2016 with two seeding dates in each year. early, E; late, L). The $p$ value of $t$ test of two genotype groups for each marker in each environment is smaller than 0.001. SV, SDS-sedimentation volume.

map, approximately $4.4 \mathrm{cM}$ away from SSR marker gwm550 reported by Patil et al. (2009). Likewise, QTL QGlu.spa-1B.2 (peak SNP: Excalibur_c50079_420) on the long arm of chromosome 1B was $6 \mathrm{cM}$ away from the QTL interval (barc181-psr162) identified by Zhang et al. (2008) and Conti et al. (2011) in durum wheat, and $0.5 \mathrm{cM}$ apart from the QTL (peak SNP: CAP8_c818_370) identified by Jernigan et al. (2018) in bread wheat. Of the two QTL reported by Roselló et al. (2018), QTL associated with marker wPt-1140 was located within QGlu.spa-2B.2 (peak SNP: Kukri_c25868_56) and another QTL associated with marker wPt-6894 within QGlu.spa2B.3 (peak SNP: Excalibur_c91034_141). There have been no reports for QTL on the short arm of chromosome $2 \mathrm{~B}$ close to QGlu.spa-2B.1 (peak SNP: RAC875_c38003_164). A QTL on the short arm of chromosome 3A reported by Roselló et al. (2018) is about $32 \mathrm{cM}$ away from QGlu.spa-3A.1 (peak SNP: RAC875_c64107_404) identified in this study, indicating that these two QTL might be different and QGlu.spa-3A.1 was a novel QTL. In addition, another QTL QGlu.spa-3A.2 (peak SNP: Excalibur_c14216_692 and wsnp_Ex_rep_c69864_68824236) on chromosome 3A was likely a novel QTL for SV.

\section{Identification of Putative Candidate Genes for Major Quantitative Trait Loci}

To predict the putative candidate genes at major QTL on chromosome $1 \mathrm{~A}$ and $1 \mathrm{~B}$ and to facilitate comparative mapping analysis, the sequences of the peak and flanking markers associated with QTL for SV were anchored to their physical location on the genome by aligning the marker sequence to the wild emmer wheat accession Zavitan (Triticum dicoccoides, WEWSeq_v.1.0; Avni et al., 2017) (Table S4 and Figure 9A) and the durum wheat $\mathrm{cv}$. Svevo assemblies (Maccaferri et al., 2019) (Table S4 and Figure 9B). The gene content in the two LOD drop of QTL region, corresponding to a region of $10.75 \mathrm{Mb}$ on $1 \mathrm{~A}$ and $11.78 \mathrm{Mb}$ on $1 \mathrm{~B}$, was searched.

Among the annotated high confidence genes, the gene TRIDC1AG047310 in proximity to SNP wsnp_Ex_c13186_20822127 (M6) on chromosome 1A of Zavitan, has five transcript splice variants. Three out of five splice variants encode a protein with three domains of HMW glutenin (Figure S5). TRIDC1AG047310.3 encodes a protein with sequence similarity with Glu-A1 (GenBank accession: ANJ03342) from wild emmer wheat accession TD-256 (T. 


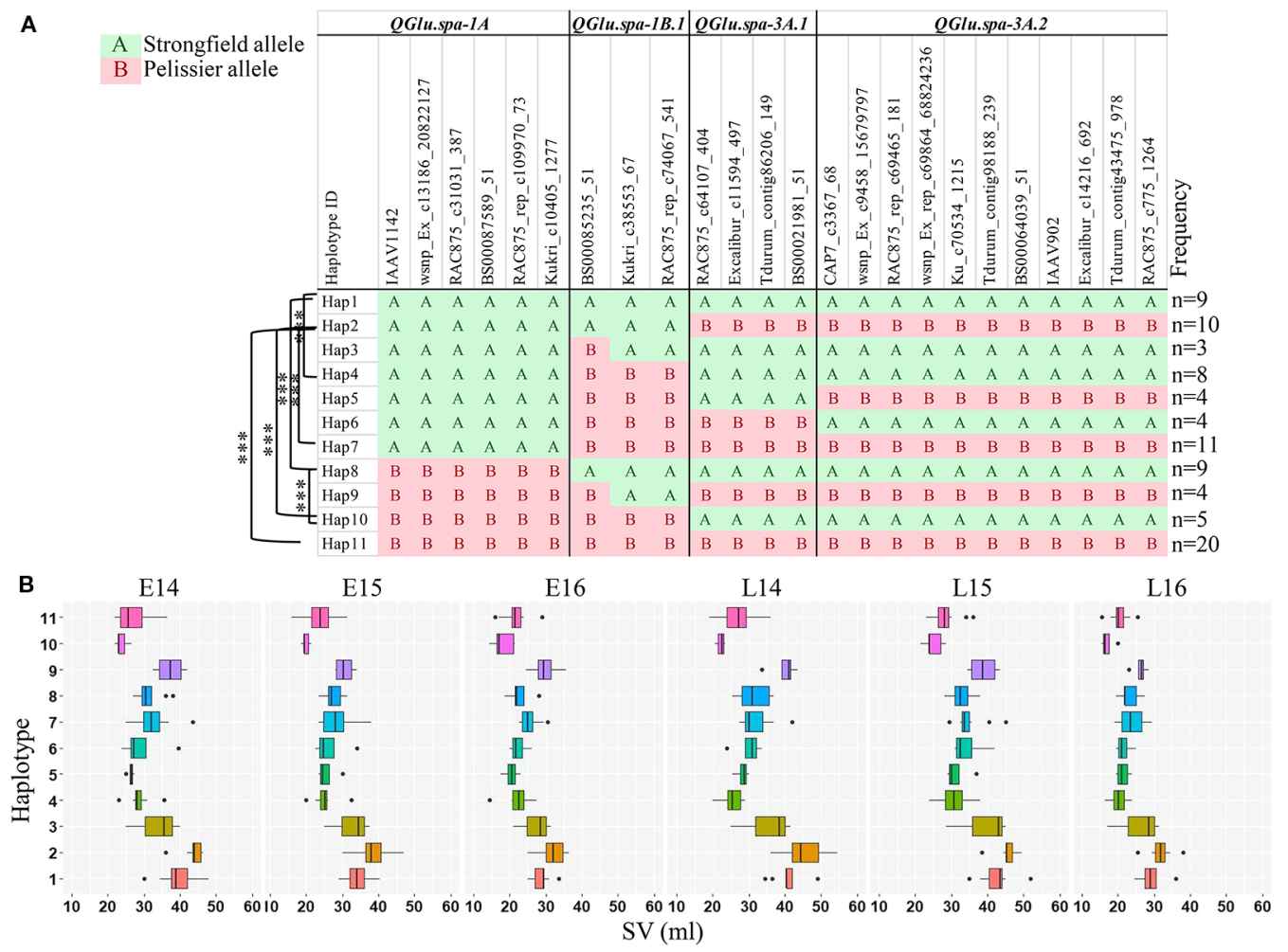

FIGURE 7 | Haplotype analysis across four quantitative trait loci (QTL) [two logarithm of odds (LOD) interval] which were identified in at least two environments. (A) Haplotype block based on single nucleotide polymorphism (SNP) markers in each QTL region. (B) Boxplots of the phenotype values corresponding to 11 different haplotype groups in each environment. Haplotypes containing less than three doubled haploid $(\mathrm{DH})$ lines were omitted from the table. The $\mathrm{DH}$ lines with undetermined haplotype were not shown. Haplotypes were assigned using R package Haplotyper. ${ }^{* \star}$, significant at $p<0.001$ ( $t$ test). SV, SDS-sedimentation volume.

TABLE 2 | Epistatic interaction between quantitative trait loci (QTL).

\begin{tabular}{|c|c|c|c|c|c|c|}
\hline QTL1 $^{\text {a }}$ & QTL2 & Environment & LOD & Effect $^{\mathrm{b}}$ & $R^{2}(\%)^{\mathrm{c}}$ & Empirical $p$-Value \\
\hline & & E15 & 2.1 & 1.8 & 1.8 & 0.015 \\
\hline & & L16 & 2.2 & 1.9 & 1.9 & 0.028 \\
\hline QGlu.spa-1A & QG/u.spa-1B.2 & L16 & 3.2 & 3.5 & 3.0 & $<0.0001$ \\
\hline QGlu.spa-1A & QG/u.spa-1B.3 & L15 & 1.6 & 1.8 & 0.8 & 0.022 \\
\hline QGlu.spa-5A & & E16 & 4.2 & 4.6 & 2.2 & $<0.0001$ \\
\hline QGlu.spa-1B.1 & QG/u.spa-6B* & E14 & 1.7 & -1.7 & 1.2 & 0.036 \\
\hline QGlu.spa-1B.4 & & L14 & 7.1 & 2.0 & 9.4 & $<0.0001$ \\
\hline QGlu.spa-1B.4 & QG/u.spa-5B* & L14 & 2.8 & -2.0 & 1.5 & $<0.0001$ \\
\hline \multirow[t]{2}{*}{ QG/u.spa-6B* } & & E14 & 0.3 & 0.4 & 0.5 & 0.777 \\
\hline & & E16 & 0.5 & 0.6 & 0.5 & 0.477 \\
\hline QGlu.spa-2B.3 & QG/u.spa-5B* & L14 & 5.0 & 6.5 & 1.8 & $<0.0001$ \\
\hline
\end{tabular}

${ }^{a} Q T L 1$ and $Q T L 2$ are a pair of interacting QTL.

${ }^{b}$ Epistatic effect of QTL1 and QTL2.

${ }^{c} R^{2}$ is the percentage of phenotypic variation explained by QTL or QTL epistasis.

*Epistatic QTL, QTL that has no or small main additive effect but statistically significant interaction effect with another QTL. 
dicoccoides) and Glu-1Ax1 (GenBank accession: CAA43331) from bread wheat (Figure S6). The HMW glutenin locus Glu-A1 reported by $\mathrm{Li}$ et al. (2009) was projected on chromosome $1 \mathrm{~A}$ where TRIDC1AG047310 is located (Figure 9A). Similarly, SNP wsnp_Ex_c13186_20822127 (M6) is about $8.66 \mathrm{Mb}$ away from Glul-A1 on chromosome 1A of durum wheat cv. Svevo (Figure
9B). This suggested that TRIDC1AG047310 might be a candidate gene underlying QTL QGlu.spa-1A. In addition, three annotated high confidence genes (TRITD1Av1G002310, TRITD1Av1G002360 and TRITD1Av1G002790) encode LMW-GS on the short arm of chromosome 1A of Svevo. However, no QTL was detected in this region in the present study.
A Durum consensus (Maccaferri 2015)

$\left.\begin{array}{l}2.6 \\ 5.7\end{array}\right]$ WPt-8770 (Giraldo et al. 2016)

10.6 BS00003595_51

14.5 - RAC875_c97040 104

22.3- wmc95 (Conti et al. 2011)

28.1 -

34.8- RAC875_C89908_105

39.9 - 17 barc148 (Zhang et al. 2008)

50.1 - BobWhite_c1488_504

60.7 gwm 357

66.9 - BM140362_603 (Conti et al. 2011)

70.5 - Kukri_c27785_400

78.0- CAP 12 C C1906_217

83.7 - wmc 312 (Conti et al. 2011)

89.5 NIAAV1142 wsnp Ex c13186 20822127

97.3. . RAC875_C31031_387

100.8 BS00073413_51

115.5 - dupw38

130.4 Kukri_c47669_204

142.1 - wPt-6754 (Roselló et al. 2018)

150.2 - RFL_Contig4538_484

167.4 - $w P t-1310 w P t-6853$

wPt-1011 (Roselló et al. 2018)

Chr1A

\section{B Durum consensus (Maccaferri 2015)}

\begin{tabular}{|c|c|}
\hline $\left.\begin{array}{r}-12.4 \\
-8.0 \\
3.9 \\
8.3 \\
19.7 \\
22.8 \\
25.1 \\
26.7 \\
28.9 \\
29.9 \\
33.0 \\
35.7 \\
36.7 \\
43.0 \\
53.4 \\
70.9 \\
76.7 \\
78.6 \\
79.6 \\
82.2 \\
82.7 \\
83.6 \\
90.7 \\
100.8 \\
109.0 \\
116.7 \\
145.4 \\
150.3 \\
155.3 \\
160.5 \\
165.6\end{array}\right]$ & 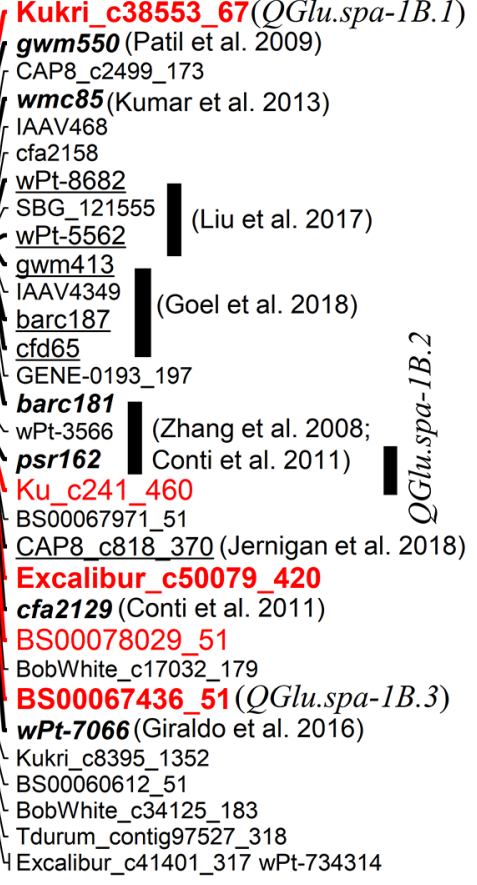 \\
\hline
\end{tabular}

\section{Pelissier $\times$ Strongfield}

119.1] BS00088136_51

120.4 IAAV1142

121.6 - wsnp Ex c13186 20822127

291 RAC875 031031387

129.8-U BS00087589_51 RAC875_rep_c109970_73

130.5 但 Kukri_c10405_1277

131.7 - BSO0022239_51 Tdurum_contig21329_326

133.0] RAC875_c6798_467

136.1 [obWhite_c23632_322

\section{Pelissier $\times$ Strongfield}

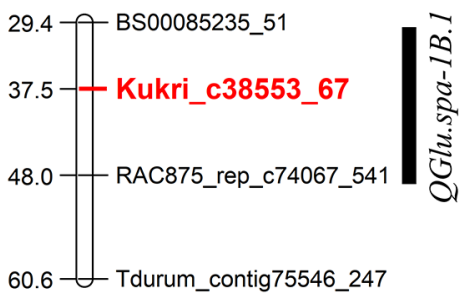

$\left.\begin{array}{l}128.1 \\ 130.0 \\ 135.4 \\ 136.1 \\ 138.6 \\ 144.1 \\ 144.8\end{array}\right\} \begin{aligned} & \text { RAC875_c1045_445 } \\ & \text { Ku_c241_460 } \\ & \text { Excalibur_c50079_420 } \\ & \text { BobWhite_c7337_717 } \\ & \text { Tdurum_contig57101_1160 } \\ & \text { BS00022551_51 } \\ & \text { BS00078029_51 }\end{aligned}$

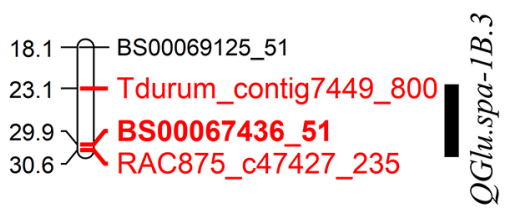

Chr1B

FIGURE 8 | Continued 
C Durum consensus (Maccaferri 2015)

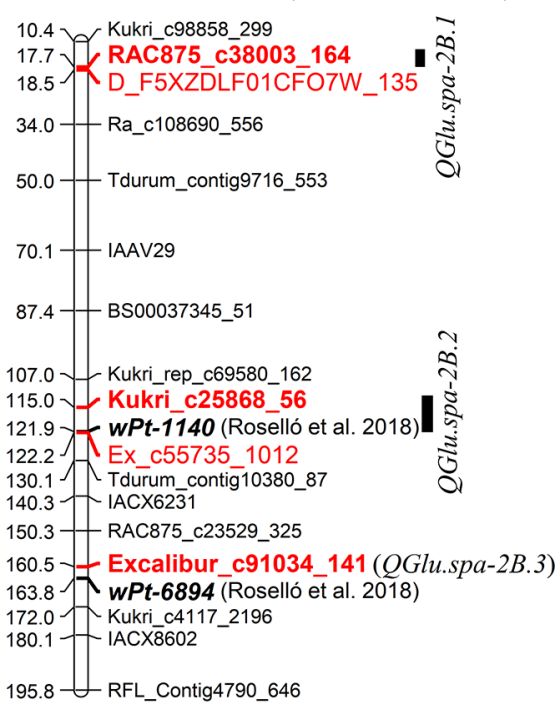

Chr2B

\section{Pelissier $\times$ Strongfield}

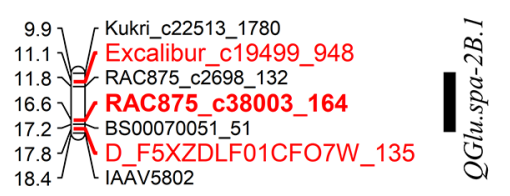

$\left.\begin{array}{l}52.7 \\ 53.3 \\ 55.9 \\ 56.5 \\ 58.3 \\ 59.0\end{array}\right]=\left[\begin{array}{l}\text { BobWhite_rep_c53204_309 } \\ \text { Excalibur_c37715_242 Kukri_c25868_56 } \\ \text { Kukri_c19751_873 } \\ \text { IAAV5226 } \\ \text { Ex_c55735_1012 } \\ \text { Ra_c72477_2165 }\end{array}\right.$

88.0 Excalibur_c33221_681

89.2 D_ Kukri_c17832_1029 RAC875_rep_c83093_126

89.9 - BS00022374-51

91.1 - wsnp_JD_c12346_12606967

93.6 Excalibur c91034 141

101.8 RAC875_c27650_216

102.4 7 - Y BobWhite rep c50285 700 Ex c66300 924

103.7 CAP7_c6910_523

D Durum consensus (Maccaferri 2015)

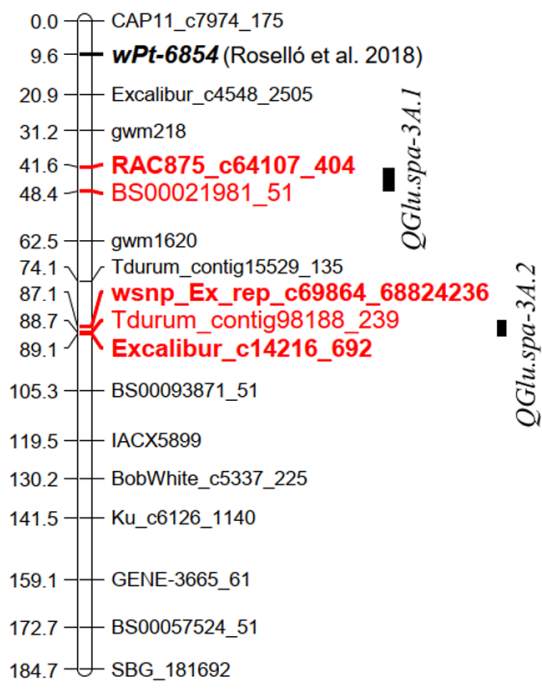

Pelissier $\times$ Strongfield
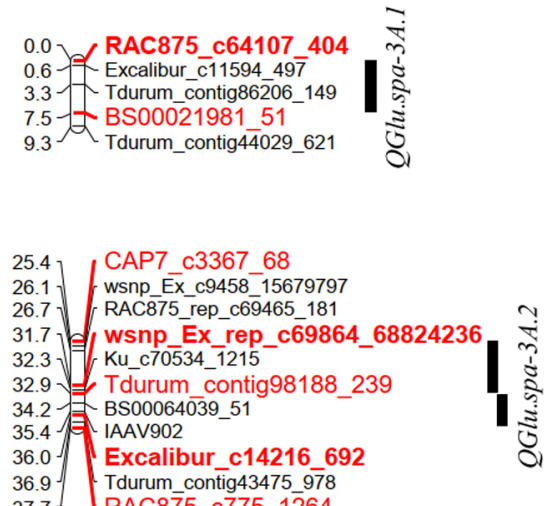

$\left.\begin{array}{l}36.9 \\ 37.7\end{array}\right]$ Tdurum_contig43475_978

Chr3A

FIGURE 8 | Projection of quantitative trait loci (QTL) for SDS-sedimentation volume (SV) reported in the literature (in both bread wheat and durum wheat) and QTL identified in this study onto the durum wheat consensus genetic map developed by Maccaferri et al. (2015) (left). The QTL on the genetic map from this present study is shown on the right. (A) Chromosome 1A, (B) 1B, (C) 2B, and (D) 3A. The markers highlighted in red and bold are peak markers of QTL identified in this study and those highlighted in red are flanking markers in two LOD drop of interval; the markers in bold and italics were reported in durum wheat; the markers underlined were reported in bread wheat.

The gene TRIDC1BG001970 on chromosome 1B of Zavitan, with a distance of $108 \mathrm{~Kb}$ from SNP Kukri_c38553_67 (M12), has three domains of gliadin/LMW glutenin. Three transcript splice variants were identified for gene TRIDC1BG001970 with TRIDC1BG001970.2 encoding a protein of 298 aa and TRIDC1BG001970.3 for a protein of 182 aa. The transcript TRIDC1BG001970.1 encodes a protein with 139 aa without
gliadin/LMW glutenin domain (Figure S7). Three paralogous genes of TRIDC1BG001970 on chromosome 1B of Zavitan were identified as TRIDC1BG001560 (1B: 7,719,618 -7,720,229 bp), TRIDC1BG001740 (1B: 8,606,652-8,608,041 bp) and TRIDC1BG004610 (1B: 20,578,493-20,579,630 bp) (Figure 9A). However, the protein structure of TRIDC1BG001970 is more similar to that of TRIDC1BG001740 (Figure S8). 
A Zavitan

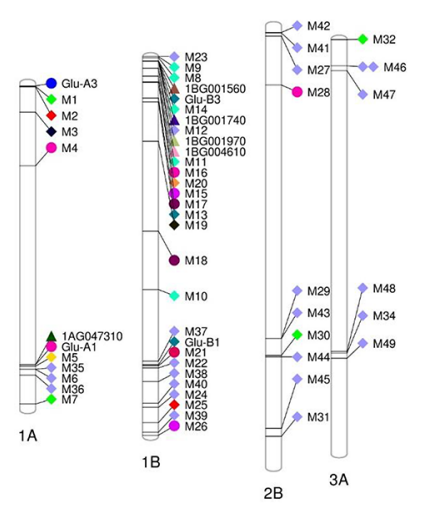

bread wheat

$\diamond$ durum
B Svevo

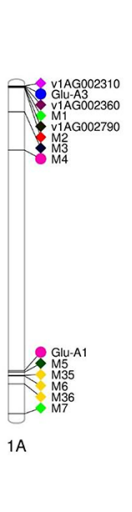

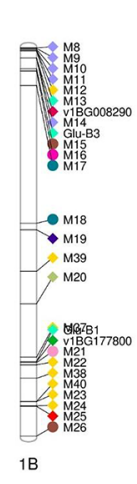

$1 \mathrm{~B}$

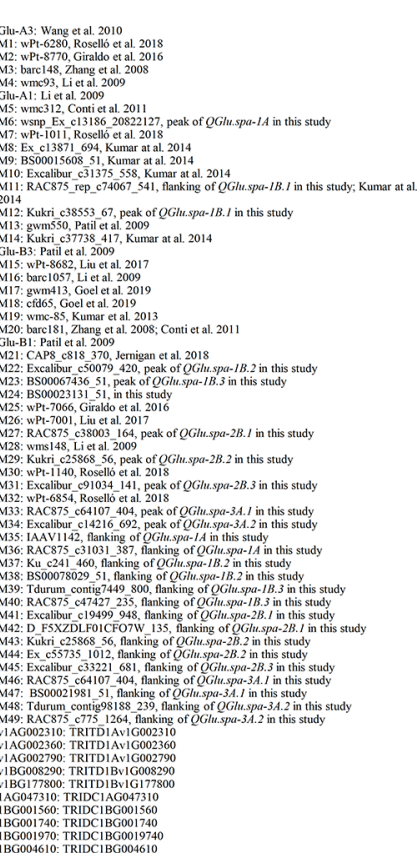

FIGURE 9 | Projection of quantitative trait loci (QTL) for SDS-sedimentation volume (SV) reported in the literature (in both bread wheat and durum wheat) and the QTL identified in this study onto the reference genomes of (A) wild emmer wheat accession Zavitan and (B) durum wheat cv. Svevo.

TRIDC1BG001970.2 shares $85 \%$ identity at the protein level with Glu-B3 (GenBank \# AVI69508.1) in durum cv. Langdon (Figure S9). On chromosome 1B of Svevo, gene TRITD1Bv1G008290 (Figure S9) encoding a portion of LMW-GS is about $105 \mathrm{~Kb}$ away from the Glu-B3 marker and $13.1 \mathrm{Mb}$ from SNP Kukri_c38553_67 (M12, QGlu.spa-1B.1). Similarly, gene TRITD1Bv1G177800 encoding a HMW-GS is $186 \mathrm{~Kb}$ away from the Glu-B1 marker and $5.88 \mathrm{Mb}$ from SNP Excalibur_c50079_420 (M22, QGlu.spa-1B.2) on the long arm of chromosome $1 \mathrm{~B}$ of Svevo. This comparative mapping indicated that TRITD1Bv1G008290 and TRITD1Bv1G177800 are likely the candidate genes for QGlu.spa-1B.1 and QGlu.spa$1 B .2$ respectively, although the possible existence of other paralogs in these regions cannot be excluded.

\section{DISCUSSION}

Gluten strength is one of the most important quality criteria in durum breeding. Previous studies have shown that gluten strength of durum wheat is quantitatively controlled by a few major QTL and some minor QTL whose expression is affected by environmental conditions. In this study, a total of nine QTL were detected for gluten strength measured by SV. Two major QTL positioned on chromosome $1 \mathrm{~A}$ and $1 \mathrm{~B}$ were detected across all environments. These two QTL together accounted for up to $59 \%$ of the phenotypic variance. The present work also allowed the identification of a few minor QTL on chromosome $1 \mathrm{~B}, 2 \mathrm{~B}$, and $3 \mathrm{~A}$, with inconsistent expression over different environments. Favorable alleles were identified from both parents at different loci.

\section{Quantitative Trait Loci Associated With Low Molecular Weight Glutenin Subunit}

The major QTL QGlu.spa-1B.1 explaining up to $40.1 \%$ of the phenotypic variance in the present study was identified on the short arm of chromosome 1B close to the LMW-GS locus Glu$B 3$. This finding supports the possibility that allelic variation for LMW-GS encoded by the Glu-B3 locus on chromosome 1BS is the major contributor for the difference of gluten strength in durum wheat (Pogna et al., 1990). A major QTL on 1BS near the Glu-B3 locus has been previously reported in a variety of durum wheat germplasm (Blanco et al., 1998; Elouafi et al., 2000; Patil et al., 2009; Kumar et al., 2013; Kumar et al., 2014), which is indicative of the importance of the $G l u-B 3$ region for gluten strength of durum wheat although with various levels of expression in different genetic backgrounds and environments. Apart from a strong positive correlation between the LMW-GS Glu-B3 locus and gluten strength, a strong association of LMWGS with pasta-cooking quality has been well documented (Pogna et al., 1990; Kovacs et al., 1995b; Ruiz and Carrillo, 1995). Similarly, nine protein alleles of the Glu-B3 (a, b, c, d, e, f, g, h, i) with various effect on dough quality have been reported in bread wheat (Metakovsky, 1990; Gupta et al., 1991).

Strongfield and Pelissier display different profiles of LMW-GS and HMW-GS (Figure S3). The analysis of allele-specific PCR markers showed that there is no polymorphism for gliadin alleles GliB1.1 and GliB1.2 between two parents, while polymorphism exists for LMW-glutenin alleles gluB3c and gluB3i (Table S5). The two characteristic subunits $(39,623$ and $42,930 \mathrm{Da}$ in Strongfield; 39,627 and 42,906 Da in Pelissier) of Glu-B3c (Wang et al., 2015) showed differential ratio in the two parents 
(Figure S3), which might result from the polymorphism of gluB3c. Combining with the variation of LMW-GS composition between parents, this indicated that Glu-B3 might be associated with QGlu.spa-1B.1 in this population. Furthermore, candidate gene analysis suggested that both TRIDC1BG001970 and TRIDC1BG001740 could be associated with QTL QGlu.spa-1B.1. The physical location of TRIDC1BG001970 and TRIDC1BG001740 is next to a major QTL (SNP: Kukri_c37738_417) contributing up to $90 \%$ of the phenotypic variation for gluten strength measured using SV in durum (Kumar et al., 2013; Kumar et al., 2014), followed by the Glu-B3 locus on the physical sequence map of wild emmer accession Zavitan. High similarity of protein structure between the identified genes and Glu-B3 suggested there could be a gene cluster in the Glu-B3 region responsible for gluten strength, which is in agreement with the report that LMW-GS are encoded by multi-gene families at the Glu-A3 and Glu-B3 loci (D'Ovidio and Masci, 2004). Further studies are needed to clone and differentiate the functions of identified genes. In addition, a new LMW-GS allele of 43,351 Da was identified in Strongfield but not in Pelissier. Further studies are required to characterize this new allele.

\section{Quantitative Trait Loci Associated With High Molecular Weight Glutenin Subunit}

In the present study, a major QTL (QGlu.spa-1A) in proximity to the HMW-GS locus Glu-A1 on chromosome 1A was detected across all environments and explained $13.7-18.3 \%$ of variation in gluten strength. Glu-A1 allele 1Ay was present in Strongfield but not in Pelissier (Table S5). The gene encoding 1Ay subunit is always silent in hexaploid wheat, while expressed in some diploid and tetraploid wheats (Jiang et al., 2009). However, no extra peak corresponding to 1 Ay was detected in Strongfield by MALDITOF-MS, which might be due to the inactivation of the $1 \mathrm{Ay}$ allele. Previous studies identified similar inactivation of $1 \mathrm{Ay}$ allele in tetraploid wheat (Jiang et al., 2009). The variation of HMW-GS subunits $1 \mathrm{Ax} 2^{\star}$ (Figure S3) is likely associated with QGlu.spa-1A, although no polymorphism was detected for allele $1 \mathrm{Ax} 2^{*}$ using PCR based analysis (Table S5). The discrepancy was most likely caused by the differential expression of the gene. In addition, the putative candidate gene for QGlu.spa-1A encodes HMW glutenin with high protein similarity to Glu-A1, suggesting that the genes associated with QGlu.spa-1A and Glu-A1 could be the same. However, the cloning of the putative candidate gene and conversion into KASP markers are required to confirm this assumption. Likewise, a QTL for gluten strength in durum wheat was detected on chromosome $1 \mathrm{AL}$, but only in one environment in a recombinant inbred line (RIL) population derived from line UC1113 and cv. Kofa (Conti et al., 2011). Another QTL associated with the HMW-GS loci detected in this study is QGlu.spa-1B.2 on the long arm of chromosome $1 \mathrm{~B}$ in a position close to $G l u-B 1$. This result agrees with the findings reported by Conti et al. (2011) that a stable QTL associated with SV was identified on chromosome 1BL (Glu$B 1)$ across multiple environments. Similarly, a QTL linked to the $G l u-B 1$ locus was also found to be associated with gluten strength in durum wheat (Patil et al., 2009). The subunit 1Bx7 (82,441 Da) detected only in Pelissier (Figure S3) is likely associated with QGlu.spa-1B.2. All these results confirmed the significant positive association between HMW-GS loci and gluten strength in durum wheat. QTL for SV associated with Glu-A1 (Li et al., 2009) and Glu-B1 (Jernigan et al., 2018) were also reported in bread wheat. However, a weaker association was reported between HMW-GS loci and gluten strength in modern durum wheat cultivars likely as a result of limited genetic variation at Glu-1 (Sissons, 2008). A weak but significant relationship between the HMW-GS and spaghetti quality was previously reported, while some studies showed no clear relationship between these two (reviewed by Liu et al., 1996). A direct measurement of rheological properties of the dough might be needed to determine the gluten strength associated with allelic variation at $G l u-A 1$ and Glu-B1 in durum wheat.

The Glu-A1 locus presented less polymorphism compared to Glu-B1 in both durum landraces and modern cultivars. In addition, the HMW-GS genes on chromosome lA were reported to have a negligible relationship with durum quality parameters when compared to the genes on chromosome $1 \mathrm{~B}$, although active Glu-A1 alleles were found to have a favorable influence on baking properties of some Italian durum [( Liu et al., 1996) and references therein]. Conti et al. (2011) identified that the most important and stable QTL for gluten strength is associated with Glu-B1 on chromosome 1BL. In contrast, in the present study, QTL associated with $G l u-A 1$ had stronger effect than the QTL at $G l u-B 1$ as evidenced by the higher percentage of phenotypic variance explained. The difference of the findings could be related to the genetic background of the parental lines used for the population development. A null allele at the Glu-A1 locus was found in Mediterranean durum wheat cultivars while non-null alleles exist in about $40 \%$ of the landraces studied (Nazco et al., 2013). Likewise, over $83 \%$ of a collection of 502 durum wheat varieties from 23 countries were found to have the GluA1c (null) allele (Branlard et al., 1989). The presence of some alleles at the Glu-B3 locus can offset the effect of the Glu-B1 alleles. Removal of the Glu-B3 effect resulted in the detection of the greatest influence of Glu-B1 (Martínez et al., 2005). In our study, the largest effect QTL (QGlu.spa-1B.1) on 1BS in the Glu-B3 region might mask the effect of Glu-B1 alleles in some environments, although no significant interaction was observed between these two loci. Further studies are necessary to confirm the assumption and elucidate the underlying mechanism.

\section{Stability of Quantitative Trait Loci}

High broad sense heritability of 0.96 was observed for SV in this study (Table S2), indicating the phenotypic variation was attributable mainly to the genetic variation. Similar high heritability value of gluten strength measured by SV has been reported in other studies carried out in durum wheat (Clarke et al., 2010; Conti et al., 2011; Kumar et al., 2013). Two stable QTL located on chromosome 1A and 1B near Glu-A1 and Glu$B 3$, respectively, were detected across all environments tested, with the trait increasing alleles derived from Strongfield. These two QTL are highly desirable for MAS as the selected favorable alleles confer high SV in all years tested and therefore are easy to 
be incorporated in breeding programs. QTL $\times \mathrm{E}$ interaction is an important contributor to the variation in the expression of complex traits. Although the genotype was the main source of variation for SV, significant QTL $\times \mathrm{E}$ were detected from the multi-trait CIM analysis of two major QTL, QGlu.spa-1A and QGlu.spa-1B.1, which were significant across all environments and displaying fluctuations in the magnitude of the effects. Another seven QTL detected on chromosome 1B, 2B, and 3A in one or two environments had favorable alleles from Pelissier. This indicates that the expression of the alleles from Pelissier is more prone to be affected by the environment and may be favored in one environment but neutral in others. As demonstrated by previous studies, gluten strength was influenced by genotype and environment, and to some extent by the interaction of genotype $\times$ environment, suggesting trials in multiple environments are required for the selection of this trait (Patil et al., 2009; Conti et al., 2011).

Furthermore, gluten strength measured by SV could be positively correlated with GPC, which depends on the genotypes and environments (Clarke et al., 2010). The moderate positive correlation between SV and GPC and weak to moderate negative correlation between SV and GY were observed in three out of six environments in this population (Figure S2). However, our studies showed that the stable QTL on $1 \mathrm{~A}$ and $1 \mathrm{~B}$ identified in this population do not contribute to GPC and GY (data unpublished).

\section{Epistatic Quantitative Trait Loci Interaction}

The identification of epistatic interactions for the QTL whose effects mostly dependent on the genotypes of other loci, can provide a more comprehensive understanding of genetic components controlling the expression of complex traits and a more accurate prediction for the phenotypic traits (Bocianowski, 2013). Of note, in the present study the epistatic interaction between QGlu.spa-1A and QGlu.spa-1B.1 was repeatedly detected in 4 out of 6 environments indicating a positive interaction between alleles of HMW-GS and LMW-GS. Therefore, it is important to take into account such epistatic effects for marker assisted selection (MAS). Significant interactions between Glu-B3 and other glutenin loci were observed in a previous study (Martínez et al., 2005). Likewise, QTL for gluten strength on 1BL was reported to have an epistatic effect with other loci having no main effect (Conti et al., 2011).

Taking together, QGlu.spa-1A and QGlu.spa-1B.1 contributed the most desirable alleles derived from parental line Strongfield and were consistently expressed over multiple environments. Two flanking markers, Kukri c38553_67 and RCA875_rep_c74067_541, in the QTL region of QGlu.spa-1B.1 can be used to effectively separate the $\mathrm{DH}$ lines into two groups with significantly different mean SV values. More distinct separation was obtained using flanking markers of both QTL QGlu.spa-1A (IAAV1142 and RAC875_c31031_387) and QGlu.spa-1B.1. The KASP assays for QGlu.spa-1A and QGlu.spa$1 B .1$ showed the good clusters and reliable results, demonstrating the effectiveness of using these KASP markers for selecting the lines with higher SV/gluten strength in durum wheat although the validation in a diverse panel is required. As such, these KASP markers have the potential to be applied for MAS in durum breeding programs. These two QTL should be subjected to map-based cloning. Although the candidate genes were predicted for these two QTL based on the QTL position on the reference genomes of durum wheat cv. Svevo and wild emmer accession Zavitan along with the comparison with previously published studies, further studies are needed to confirm our assumption. Haplotype analysis of these two QTL along with another two QTL on 3A indicated the DH lines with the combination of all favorable alleles at each QTL had the highest mean SV across all environments. These DH lines are the good candidates as parental lines for developing new varieties with strong gluten strength. Similar haplotype analysis of QTLQGlu.spa-1A and QGlu.spa-1B.1 in a diverse durum panel will enhance our understanding of the allelic variants of $G l u-A 1$ and $G l u-B 3$ and may facilitate more effective use of favorable alleles in further improving gluten strength of durum wheat.

\section{DATA AVAILABILITY STATEMENT}

All datasets generated for this study are included in the article/ Supplementary Material.

\section{AUTHOR CONTRIBUTIONS}

YR and RK conceptualized this study. YR, RK, AS, RD and RC generated the population and implemented the field trials and phenotyping of the population. IP and PG conducted the marker validation and the KASP assays. WZ, AS and PF provided the genotyping platform. BY conducted data analysis. BY and YR contributed to data management and visualization. BY wrote the original manuscript and $\mathrm{YR}, \mathrm{RK}, \mathrm{RD}$, and PF contributed to the review and editing of the manuscript. YR was the principal investigator and acquired the fund for this study.

\section{FUNDING}

Financial support was received from the National Wheat Improvement Program and the Canadian Agricultural Partnership with support from Agriculture and Agri-Food Canada, Western Grains Research Foundation, Alberta Wheat Commission, Saskatchewan Wheat Development Commission, and Manitoba Wheat and Barley Growers Association. The work was also supported by Canadian Wheat Improvement Flagship funded by National Research Council Canada.

\section{ACKNOWLEDGMENTS}

We gratefully acknowledge the support of Agriculture and Agrifood Canada (AAFC), Western Grain Research Foundation (WGRF), National Research Council Canada (NRC), Alberta Wheat Commission, Saskatchewan Wheat Development Commission, and Manitoba Wheat and Barley Growers Association in carrying out this study. We thank Christine Sidebottom and Janet Condie at NRC for technical assistance 
with DNA extraction and genotyping using the Infinium iSelect Wheat 90K SNP chip. The technical support of Wheat Breeding Group at the Swift Current Research and Development Centre and Cereal Quality Lab in Winnipeg from AAFC is sincerely appreciated.

\section{REFERENCES}

Ames, N. P., Clarke, J. M., Marchylo, B. A., Dexter, J. E., Schlichting, L. M., and Woods, S. M. (2003). The effect of extra-strong gluten on quality parameters in durum wheat. Can. J. Plant Sci. 83, 525-532. doi: 10.4141/P02-072

Anderson, O. D., Gu, Y. Q., Kong, X., Lazo, G. R., and Wu, J. (2009). The wheat $\omega-$ gliadin genes: structure and EST analysis. Funct. Integr. Genomics 9, 397-410. doi: 10.1007/s10142-009-0122-2

Autran, J. C., and Feillet, P. (1985). "Genetic and technological basis of protein quality for durum wheat in pasta," in Protein Evaluation in Cereals and Legumes. Report EUR 10404. Ed. A. Pattakou (Luxemburg: Commission of the European Communities), 59-71.

Avni, R., Nave, M., Barad, O., Baruch, K., Twardziok, S. O., Gundlach, H., et al. (2017). Wild emmer genome architecture and diversity elucidate wheat evolution and domestication. Science 97, 93-97. doi: 10.1126/science.an0032

Barak, S., Mudgil, D., and Khatkar, B. S. (2015). Biochemical and functional properties of wheat gliadins: a review. Crit. Rev. Food Sci. 55, 357-368. doi: 10.1080/10408398.2012.654863

Blanco, A., Bellomo, M. P., Lotti, C., Maniglio, T., Pasqualone, A., Simeone, R., et al. (1998). Genetic mapping of sedimentation volume across environments using recombinant inbred lines of durum wheat. Plant Breed. 117, 413-417. doi: 10.1111/j.1439-0523.1998.tb01965.x

Bocianowski, J. (2013). Epistasis interaction of QTL effects as a genetic parameter influencing estimation of the genetic additive effect. Genet. Mol. Biol. 36, 93100. doi: 10.1590/S1415-47572013000100013

Branlard, G., Autran, J. C., and Monneveaux, P. (1989). High molecular weight glutenin subunits of durum wheat (T. durum). Theor. Appl. Genet. 78, 353-358. doi: $10.1007 /$ BF00265296

Brites, C., and Carrillo, J. M. (2001). Influence of high molecular weight (HMW) and low molecular weight (LMW) glutenin subunits controlled by Glu-1 and Glu-3 loci on durum wheat quality. Cereal Chem. 78, 59-63. doi: 10.1094/ CCHEM.2001.78.1.59

Broman, K. W., Wu, H., Sen, Ś., and Churchill, G. A. (2003). R/qtl: QTL mapping in experimental crosses. Bioinformatics 19, 889-890. doi: 10.1093/ bioinformatics/btg112

Cavanagh, C. R., Chao, S. M., Wang, S. C., Huang, B. E., Stephen, S., Kiani, S., et al. (2013). Genome-wide comparative diversity uncovers multiple targets of selection for improvement in hexaploid wheat landraces and cultivars. Proc. Natl. Acad. Sci. U.S.A. 110, 8057-8062. doi: 10.1073/pnas.1217133110

Clarke, J. M., Marchylo, B., Kovac, M. I. P., Noll, J. S., McCaig, T. N., and Howes, N. K. (1998). Breeding durum wheat for pasta quality in Canada. Euphytica 100, 163-170. doi: 10.1023/A:1018313603344

Clarke, J. M., McCaig, T. N., DePauw., R. M., Knox, R. E., Clarke, F. R., Fernandez, M. R., et al. (2005). Strongfield durum wheat. Can. J. Plant Sci. 85, 651-654. doi: 10.4141/P04-119

Clarke, F. R., Clarke, J. M., Ames, N. A., Knox, R. E., and Ross, J. R. (2010). Gluten index compared with SDS-sedimentation volume for early generation selection for gluten strength in durum wheat. Can. J. Plant Sci. 90, 1-11. doi: 10.4141/ CJPS09035

Conti, V., Roncallo, P. F., Beaufort, V., Cervigni, G. L., Miranda, R., Jensen, C. A., et al. (2011). Mapping of main and epistatic effect QTLs associated to grain protein and gluten strength using a RIL population of durum wheat. J. Appl. Genet. 52, 287-298. doi: 10.1007/s13353-011-0045-1

D'Ovidio, R., and Masci, S. (2004). The low-molecular-weight glutenin subunits of wheat gluten. J. Cereal Sci. 39, 321-339. doi: 10.1016/j.jcs.2003.12.002

Dexter, J. E., Matsuo, R. R., Kosmolak, F. G., Leisle, D., and Marchylo, B. A. (1980). The suitability of the SDS-sedimentation test for assessing gluten strength in durum wheat. Can. J. Plant Sci. 60, 25-29. doi: 10.4141/cjps80-004

Dexter, J. E. (2008). The history of durum wheat breeding in Canada and summaries of recent research at the Canadian Grain Commission on factors

\section{SUPPLEMENTARY MATERIAL}

The Supplementary Material for this article can be found online at: https://www.frontiersin.org/articles/10.3389/fpls.2020.00170/ full\#supplementary-material

associated with durum wheat processing, in Bosphorus 2008 ICC (Istanbul: International Cereal Congress).

Dick, J. W., and Quick, J. S. (1983). A modified screening test for rapid estimation of gluten strength in early generation durum wheat breeding. Cereal Chem. 60, 315-318.

Du Cros, D. L. (1987). Gluten proteins and gluten strength in durum wheat. J. Cereal Sci. 5, 3-12. doi: 10.1016/S0733-5210(87)80003-6

Elfatih, S., Peng, Y., Ma, J., Peng, J., Sun, D., and Ma, W. (2013). High frequency of unusual high molecular weight glutenin alleles in 232 tetraploid durum wheat accessions (Triticum turgidumL. ssp. durum Desf). Cereal Res. Commun. 41, 583-592. doi: 10.1556/CRC.2013.0031

Elouafi, I., Nachit, M., Elsaleh, A., Asbati, A., and Mather, D. E. (2000). "QTLmapping of genomic regions controlling gluten strength in durum (Triticum turgidum L. var. durum)," in Durum Wheat Improvement in The Mediterranean Region: New Challenges. Eds. C. Royo, M. Nachit, N. Di Fonzo and J. L. Araus (Zaragoza: CIHEAM), 505-509.

Feillet, P., Ait-Mouh, O., Kobrehel, K., and Autran, J. C. (1989). The role of low molecular weight glutenin proteins in the determination of cooking quality of pasta products: an overview. Cereal Chem. 66, 26-30.

Frutos, E., Galindo, M. P., and Leiva, V. (2014). An interactive biplot implementation in $\mathrm{R}$ for modeling genotype-by-environment interaction. Stoch Environ. Res. Risk Assess. 28, 1629-1641. doi: 10.1007/s00477-0130821-Z

Giraldo, P., Royo, C., González, M., Carrillo, J. M., and Ruiz, M. (2016). Genetic diversity and association mapping for agromorphological and grain quality traits of a structured collection of durum wheat landraces including subsp. durum, turgidum and diccocon. PloS One 11, e0166577. doi: 10.1371/ journal.pone.0166577

Goel, S., Singh, K., Singh, B., Grewa, S., Dwivedi, N., Alqarawi, A. A., et al. (2019). Analysis of genetic control and QTL mapping of essential wheat grain quality traits in a recombinant inbred population. PloS One. 14, e0200669. doi: 10.1371/journal.pone.0200669

Gupta, R. B., Bekes, F., and Wrigley, C. W. (1991). Prediction of physical dough properties from glutenin subunit composition in bread wheats. Cereal Chem. $68,328-333$.

Heffelfinger, C., Fragoso, C. A., and Lorieux, M. (2017). Constructing linkage maps in the genomics era with MapDisto 2.0. Bioinformatics. 33, 2224-2225. doi: 10.1093/bioinformatics/btx177

Henkrar, F., El-Haddoury, J., Iraqi, D., Bendaou, N., and Udupa, S. M. (2017). Allelic variation at high-molecular weight and low-molecular weight glutenin subunit genes in Moroccan bread wheat and durum wheat cultivars. 3 Biotech. 7, 287. doi: 10.1007/s13205-017-0908-1

Humphreys, G. D., and Knox, R. E. (2015). "Doubled-Haploid Breeding in Cereals," in Advances in Plant Breeding Strategies: Breeding, Biotechnology and Molecular Tools. Eds. J. M. Al-Khayri, S. M. Jain and D. V. Johnson (Switzerland: Springer International Publishing). doi: 10.1007/978-3-31922521-0_9

Irvine, G. N. (1971). "Durum wheat and paste products," in Wheat: Chemistry and Technology, vol. III . Ed. Y. Promeranz (St. Paul, Minn: American Association of Cereal Chemists), 777-798.

Jernigan, K. L., Godoy, J. V., Huang, M., Zhou, Y., Morris, C. F., GarlandCampbell, K. A., et al. (2018). Genetic dissection of end-use quality traits in adapted soft white winter wheat. Front. Plant Sci. 9, 271. doi: 10.3389/ fpls.2018.00271

Jiang, Q., Wei, Y., Wang, F., Wang, J.-R., Yan, Z.-H., and Zheng, Y.-L. (2009). Characterization and comparative analysis of HMW glutenin 1Ay alleles with differential expressions. BMC Plant Biol. 9, 16. doi: 10.1186/1471-2229-9-16

Joppa, L. R., Khan, K., and Williams, N. D. (1983). Chromosomal location of genes for gliadin polypeptides in durum wheat Triticum turgidum. Theor. Appl. Genet. 64, 289-293. doi: 10.1007/BF00274164 
Kovacs, M. I. P., Howes, N. K., Leisle, D., and Skerritt, J. H. (1991). "The Effect of High Molecular Weight Glutenin Subunit Composition on Tests Used to Predict Durum Wheat Quality," in Cereals Int. Proc. Victoria. Eds. D. F. Martin and C. W. Wrigley (Melbourne, Australia: Royal Australian Chemical Institute), 257-262.

Kovacs, M. I. P., Howes, N. K., Leisle, D., and Skerritt, J. H. (1993). The effect of high $\mathrm{Mr}$, glutenin subunit composition on the results from tests used to predict durum wheat quality. J. Cereal Sci. 18, 43-51. doi: 10.1006/jcrs.1993.1032

Kovacs, M. I. P., Noll, J. S., Dahlke, G., and Leisle, D. (1995a). Pasta viscoelasticity: its usefulness in the Canadian durum wheat breeding program. J. Cereal Sci. 22, 115-121. doi: 10.1016/0733-5210(95)90040-3

Kovacs, M. I. P., Howes, N. K., Leisle, D., and Zawistowski, J. (1995b). Effect of two different low molecular weight glutenin subunits on durum wheat pasta quality parameters. Cereal Chem. 72, 85-87.

Kovacs, M. I. P., Poste, L. M., Butler, G., Woods, S. M., Leisle, D., Noll, J. S., et al. (1997). Durum wheat quality: comparison of chemical and rheological screening tests with sensory analysis. J. Cereal Sci. 25, 65-75. doi: 10.1006/jcrs.1996.0069

Kumar, A., Jain, S., Iqbal, M. J., Elias, E. M., Kianian, S., and Mergoum, M.Fine Mapping and Validation of a Major QTL for Gluten Strength, in: ASA, CSSA \& SSSA International Annual Meeting, Long Beach, CA., Nov. 2-5, 2014.

Kumar, A., Elias, E. M., Ghavami, F., Xu, X., Jain, S., Manthey, F. A., et al. (2013). A major QTL for gluten strength in durum wheat (Triticum turgidum L. var. durum). J. Cereal Sci. 57, 21-29. doi: 10.1016/j.jcs.2012.09.006

Laurie, C., Wang, S., Carlini-Garcia, L. A., and Zeng, Z. B. (2014). Mapping epistatic quantitative trait loci. BMC Genet. 15, 112. doi: 10.1186/s12863-014-0112-9

Li, Q. Y., Yan, Y. M., Wang, A. L., An, X. L., Zhang, Y. Z., Hsam, S. L. K., et al. (2006). Detection of HMW glutenin subunit variations among 205 cultivated emmer accessions (Triticum turgidum spp. dicoccum). Plant Breed. 125, 120124. doi: 10.1111/j.1439-0523.2006.01173.x

Li, Y., Song, Y., Zhou, R., Branlard, G., and Jia, J. (2009). Detection of QTLs for bread-making quality in wheat using a recombinant inbred line population. Plant Breed. 128, 235-243. doi: 10.1111/j.1439-0523.2008.01578.x

Liu, C. Y., Shepherd, K. W., and Rathjen, A. J. (1996). Improvement of durum wheat pasta making and bread making qualities. Cereal Chem. 73, 155-166.

Liu, T.-T., Liu, K., Wang, F.-F., Zhang, Y., Li, Q.-F., Zhang, K.-R., et al. (2017). Conditional and unconditional QTLs mapping of gluten strength in common wheat (Triticum aestivum L.). J. Integr. Agr. 16, 2145-2155. doi: 10.1016/ S2095-3119(16)61564-2

Maccaferri, M., Sanguineti, M. C., Corneti, S., Ortega, J. L., Salem, M. B., Bort, J., et al. (2008). Quantitative trait loci for grain yield and adaptation of durum wheat (Triticum durum Desf.) across a wide range of water availability. Genetics 178, 489-511. doi: 10.1534/genetics.107.077297

Maccaferri, M., Ricci, A., Salvi, S., Milner, S. G., Noli, E., Martelli, P. L., et al. (2015). A high-density, SNP-based consensus map of tetraploid wheat as a bridge to integrate durum and bread wheat genomics and breeding. Plant Biotechnol. J. 13, 648-663. doi: 10.1111/pbi.12288

Maccaferri, M., Harris, N. S., Twardziok, S. O., Pasam, R. K., Gundlach, H., Spannagl, M., et al. (2019). Durum wheat genome highlights past domestication signatures and future improvement targets. Nat. Genet. 51, 885-895. doi: 10.1038/s41588-019-0381-3

Martínez, M. C., Ruiz, M., and Carrillo, J. M. (2005). Effects of different prolamin alleles on durum wheat quality properties. J. Cereal Sci. 41, 123-131. doi: 10.1016/j.jcs.2004.10.005

Metakovsky, E. V., Wrigley, C. W., Bekes, F., and Gupta, R. B. (1990). Gluten polypeptides as useful genetic markers of dough quality in Australian wheats. Aust. J. Agric. Res. 41, 289-306. doi: 10.1071/AR9900289

N'Diaye, A., Haile, J. K., Nilsen, K. T., Walkowiak, S., Ruan, Y., Singh, A. K., et al. (2018). Haplotype loci under selection in Canadian durum wheat germplasm over 60 years of breeding: association with grain yield, quality traits, protein loss, and plant height. Front. Plant Sci. 9, 1589. doi: 10.3389/fpls.2018.01589

Nazco, R., Peña, R. J., Ammar, K., Villegas, D., Crossa, J., Moragues, M., et al. (2013). Variability in glutenin subunit composition of Mediterranean durum wheat germplasm and its relationship with gluten strength. J. Agric. Sci. 152, 379-393. doi: 10.1017/S0021859613000117

Pasqualone, A., Piarulli, L., Mangini, G., Gadaleta, A., Blanco, A., and Simeone, R. (2015). Quality characteristics of parental lines of wheat mapping populations. Agric. Food Sc. 24, 118-127. doi: 10.23986/afsci.49570

Patil, R. M., Oak, M. D., Tamhankar, S. A., and Rao, V. S. (2009). Molecular mapping of QTLs for gluten strength as measured by sedimentation volume and mixograph in durum wheat (Triticum turgidum L. ssp durum). J. Cereal Sci. 49, 378-386. doi: 10.1016/j.jcs.2009.01.001

Payne, P. I., and Lawrence, G. J. (1983). Catalogue of alleles for the complex loci, Glu-A1, Glu-B1 and Glu-D1 which code for high-molecular-weight subunits of glutenin in hexaploid wheat. Cereal Res. Commun. 11, 29-35.

Payne, P. I. (1987). Genetics of wheat storage proteins and the effect of allelic variation on bread-making quality. Ann. Rev. Plant Physiol. 38, 141-153. doi: 10.1146/annurev.pp.38.060187.001041

Pogna, N. E., Autran, J. C., Mellini, F., Lafiandra, D., and Feillet, P. (1990). Chromosome 1B-encoded gliadins and glutenin subunits in durum wheat: genetics and relationship to gluten strength. J. Cereal Sci. 11, 15-34. doi: 10.1016/S0733-5210(09)80178-1

Quick, J. S., and Donnelly, B. J. (1980). A rapid test for estimating durum wheat gluten quality. Crop Sci. 20, 816-818. doi: 10.2135/cropsci1980.0011183X002000060037x

Rasheed, A., Wen, W., Gao, F., Zhai, S., Jin, H., Liu, J., et al. (2016). Development and validation of KASP assays for genes underpinning key economic traits in bread wheat. Theor. Appl. Genet. 129, 1843-1860. doi: 10.1007/s00122-016-2743-x

Roselló, M., Royo, C., Álvaro, F., Villegas, D., Nazco, R., and Soriano, J. M. (2018). Pasta-making quality QTLome from mediterranean durum wheat landraces. Front. Plant Sci. 9, 1512. doi: 10.3389/fpls.2018.01512

Ruiz, M., and Carrillo, J. M. (1995). Separate effects on gluten strength of Gli-1 and Glu-3 prolamin genes on chromosomes 1A and 1B in durum wheat. J. Cereal Sci. 21, 137-144. doi: 10.1016/0733-5210(95)90029-2

Sapirstein, H. D., David, P., Preston, K. R., and Dexter, J. E. (2007). Durum wheat breadmaking quality: effects of gluten strength, protein composition, semolina particle size and fermentation time. J. Cereal Sci. 45, 150-161. doi: 10.1016/ j.jcs.2006.08.006

Shewry, P. R., Halford, N. G., and Tatham, A. S. (1992). The high molecular weight subunits of wheat glutenin. J. Cereal Sci. 15, 105-120. doi: 10.1016/S0733-5210 (09)80062-3

Sissons, M. J., Soh, H. N., and Turner, M. A. (2007). Role of gluten and its components in influencing durum wheat dough properties and spaghetti cooking quality. J. Sci. Food Agric. 87, 1874-1885. doi: 10.1002/jsfa.2915

Sissons, M. (2008). Role of durum wheat composition on the quality of pasta and bread. Food 2, 75-90.

Voorrips, R. E. (2002). MapChart: Software for the graphical presentation of linkage maps and QTLs. J. Hered. 93, 77-78. doi: 10.1093/jhered/93.1.77

Wang, L. H., Li, G. Y., Peña, R. J., Xia, X. C., and He, Z. H. (2010). Development of STS markers and establishment of multiplex PCR for Glu-A3 alleles in common wheat (Triticum aestivum L.). J. Cereal Sci. 51, 305-312. doi: 10.1016/j.jcs.2010.01.005

Wang, S., Basten, C. J., and Zeng, Z.-B. (2012). Windows QTL Cartographer 2.5. Department of Statistics (Raleigh, NC: North Carolina State University). (http://statgen.ncsu.edu/qtlcart/WQTLCart.htm).

Wang, A., Liu, L., Peng, Y., Islam, S., Applebee, M., Appels, R., et al. (2015). Identification of low molecular weight glutenin alleles by matrix-assisted laser desorption/ionization time-of-flight mass spectrometry (MALDI-TOF-MS) in common wheat (Triticum aestivum L.). PloS One 10 (9), e0138981. doi: 10.1371/ journal.pone.0138981

Wrigley, C., Bekes, F., and Bushuk, W. (2006). "Gliadin and Glutenin," in The Unique Balance of Wheat Quality, 1st Ed (MN: AACC International), 3-32.

Xu, L. L., Li, W., Wei, Y. M., and Zheng, Y. L. (2009). Genetic diversity of HMW glutenin subunits in diploid, tetraploid and hexaploid Triticum species. Genet. Resour. Crop Evol. 56, 377-391. doi: 10.1007/s10722-008-9373-3

Zhang, W., Chao, S., Manthey, F., Chicaiza, O., Brevis, J. C., Echenique, V., et al. (2008). QTL analysis of pasta quality using a composite microsatellite and SNP map of durum wheat. Theor. Appl. Genet. 117, 1361-1377. doi: 10.1007/s00122-008-0869-1

Conflict of Interest: The authors declare that the research was conducted in the absence of any commercial or financial relationships that could be construed as a potential conflict of interest.

Copyright $\odot 2020$ Ruan, Yu, Knox, Singh, DePauw, Cuthbert, Zhang, Piche, Gao, Sharpe and Fobert. This is an open-access article distributed under the terms of the Creative Commons Attribution License (CC BY). The use, distribution or reproduction in other forums is permitted, provided the original author(s) and the copyright owner(s) are credited and that the original publication in this journal is cited, in accordance with accepted academic practice. No use, distribution or reproduction is permitted which does not comply with these terms. 